Engineering Physics and Mathematics Division

Mathematical Sciences Section

\title{
A NEW SHARED-MEMORY PROGRAMMING PARADIGM FOR MOLECULAR DYNAMICS SIMULATIONS ON THE INTEL PARAGON
}

\author{
E.F. D'Azevedo \\ C.H. Romine \\ Mathematical Sciences Section \\ Oak Ridge National Laboratory \\ P.O. Box 2008, Bldg. 6012 \\ Oak Ridge, TN 37831-6367
}

Date Published: March 1995

Research supported by the Applied Mathematical Sciences subprogram of the Office of Energy Research, U.S. Department of Energy

\author{
Prepared by the \\ Oak Ridge National Laboratory \\ Oak Ridge, Tennessee 37831 \\ managed by \\ Martin Marietta Energy Systems, Inc. \\ for the \\ U.S. DEPARTMENT OF ENERGY \\ under Contract No. DE-AC05-84OR21400
}




\section{Contents}

1 Introduction ...................... 1

1.1 DOLIB (Distributed Object Library) $\ldots \ldots \ldots \ldots \ldots \ldots$

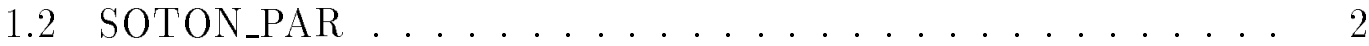

2 Overview of Parallelization of SOTON_PAR with DOLIB . . . . . . 3

2.1 Geometric Hashing . . . . . . . . . . . . . . . 3

2.2 DOLIB implementation of movout . . . . . . . . . . 4

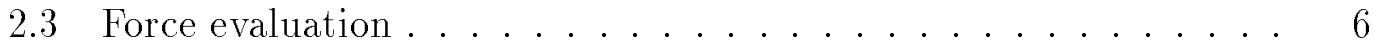

2.4 Dynamic Load Balancing . . . . . . . . . . . . . . . 7

2.5 Shifted Force Potential . . . . . . . . . . . . . . 8

3 Parallel Performance . . . . . . . . . . . . . . . 8

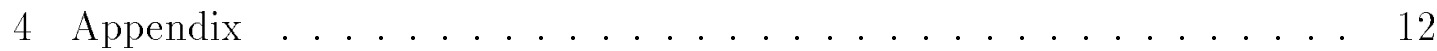

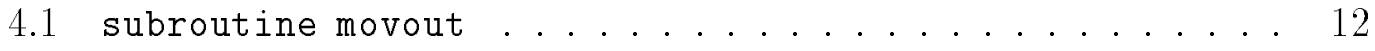

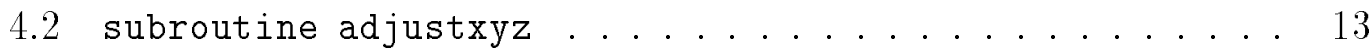

4.3 subroutine hashxyz . . . . . . . . . . . . . 15

4.4 subroutine setxlist . . . . . . . . . . . . . 19

4.5 subroutine setuplist . . . . . . . . . . . 22

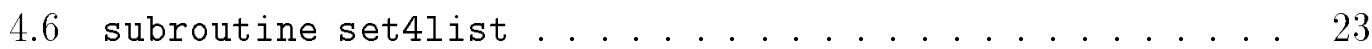

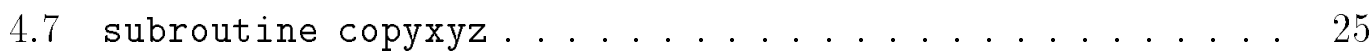

4.8 subroutine force . . . . . . . . . . . . . . . 29

4.9 subroutine forceall . . . . . . . . . . . . . . 31

4.10 subroutine forcecol . . . . . . . . . . . . 33

4.11 subroutine forcecal . . . . . . . . . . . . . . 45

5 References ..................... 51 


\section{List of Figures}

2.1 Top view of columns used in processing column $(i, j)$. Only two new columns are needed in processing column $(i+1, j) \ldots \ldots 7$ 


\title{
A NEW SHARED-MEMORY PROGRAMMING PARADIGM FOR MOLECULAR DYNAMICS SIMULATIONS ON THE INTEL PARAGON
}

\author{
E.F. D'Azevedo \\ C.H. Romine
}

\begin{abstract}
This report describes the use of shared memory emulation with DOLIB (Distributed Object Library) to simplify parallel programming on the Intel Paragon. A molecular dynamics application is used as an example to illustrate the use of the DOLIB shared memory library. SOTON_PAR, a parallel molecular dynamics code with explicit message-passing using a Lennard-Jones 6-12 potential, is rewritten using DOLIB primitives. The resulting code has no explicit message primitives and resembles a serial code. The new code can perform dynamic load balancing and achieves better performance than the original parallel code with explicit messagepassing.
\end{abstract}




\section{Introduction}

This report describes the use of shared memory emulation provided by DOLIB (Distributed Object Library) [3] for parallel programming of large-scale Molecular Dynamics (MD) codes on the Intel Paragon distributed memory supercomputer. SOTON_PAR [7], a parallel MD code that uses explicit message passing and Lennard-Jones atoms (6-12 potential), is rewritten using DOLIB shared memory primitives. The new MD prototype code can perform dynamic load balancing and achieves better performance than the original code that uses spatial decomposition. This report may also serve as a guide for scientists or programmers considering the use of DOLIB shared memory emulation in more sophisticated molecular dynamics simulations or particle-in-cell methods.

While this prototype code uses a simple Lennard-Jones 6-12 potential, more complicated force evaluation (such as including angular (three-body) forces for silicon and torsional (four-body) forces for organic polymers or proteins) can easily be added using the DOLIB shared memory framework. DOLIB's dynamic load balancing capability is especially attractive for more demanding MD simulations.

\subsection{DOLIB (Distributed Object Library)}

DOLIB (Distributed Object Library) is a set of FORTRAN and $\mathrm{C}$ callable routines to emulate shared memory on distributed-memory environments such as Intel multiprocessors and PVM clusters of workstations.

DOLIB supports runtime dynamic creation and destruction of one-dimensional arrays. Explicit gather and scatter operations provide access to array elements. DOLIB is portable since no language extension is introduced and no preprocessor, compiler or operating system support is required. DOLIB provides an atomic accumulate operation axpby $(y($ index $(:) \leftarrow \alpha * x(:)+\beta * y($ index $(:))$, where $x$ is a local array, $y$ is a globally shared array, and index is an array of indices for $y$. axpby is intended for use in finite element matrix assembly, with no need for explicit lock/unlock. Note that $\alpha=1, \beta=0$ corresponds to a scatter operation. DOLIB also provides automatic caching of read-only data to reduce 
message traffic. However, the programmer has the responsibility of flushing the cache to maintain coherency during concurrent updates.

A global array in DOLIB is stored as fixed-size pages in a block wrapped mapped fashion across all processors. DOLIB translates requests for remote data (gather) or update (scatter) into the appropriate message sent to the "owner" processor of that data page. These DOLIB requests are then serviced by the IPX [6] message system.

DOLIB supports a generalized atomic update operation (axpbyz),

$$
\begin{aligned}
z(:) & \leftarrow y(\operatorname{index}(:)) \\
y(\operatorname{index}(:)) & \leftarrow \alpha * x(:)+\beta * y(\operatorname{index}(:)) \\
x(:) & \leftarrow z(:)
\end{aligned}
$$

Operation axpbyz can also be used to implement a vector of counting semaphores.

\subsection{SOTON_PAR}

The original version of SOTON_PAR ${ }^{1}$ MD code ran on the Intel iPsc/2. The version we obtained was substantially modified by David Walker to run efficiently on Intel Paragon multiprocessors. SOTON_PAR models short range inter-atomic interactions by using a link-cell (geometric hashing) algorithm where all particles are hashed into three-dimensional $N_{b} \times N_{b} \times N_{b}$ bins. The bin size is at least the cut-off distance $\left(r_{c}\right)$ used in the short-range force evaluation. SOTON_PAR exploits the symmetry of Newton's Third Law by examining atoms in only 13 (instead of 26) neighboring cells. It uses a spatial decomposition [8] and requires message exchanges with neighbor processors at each time step as atoms migrate across processor boundaries. Many of the MD algorithms used in SOTON_PAR are described in the book Computer Simulation of Liquids [1]. The code also uses a "shifted-force" [9] Lennard-Jones 6-12 potential to avoid a discontinuity at the

\footnotetext{
${ }^{1}$ The source code for SOTON_PAR is available from the CCP5 archive at ftp://ftp.dl.ac.uk/ccp5/SOTON_PAR/mdplli3cu.master
} 
cut-off distance.

The code consists of two parts: the "host" code runs on the service partition and controls the I/O, allocation and loading of nodes etc.; the "worker" code runs on the compute nodes and performs most of the work in geometric hashing and force evaluations.

The code assumes an $N_{c} \times N_{c} \times N_{c}$ FCC (Face Centered Cubic) lattice scaled to the canonical unit cube $[-0.5,0.5] \times[-0.5,0.5] \times[-0.5,0.5]$, with a total of $N=4 N_{c}^{3}$ atoms. A periodic boundary condition is imposed on all sides. A simple Verlet leap-frog time stepping scheme is used to update the particle positions.

\section{Overview of Parallelization of SOTON_PAR with DOLIB}

We have written a new parallel MD prototype code based on SOTON_PAR using DOLIB shared memory primitives. The new code shares much of the overall structure of SOTON_PAR with most of the host code for performing I/O executed by node 0 . We believe the new code is much easier to write and understand without the complexities of explicit message passing code. We have also added several enhancements: (1) initialize with Boltzmann distribution for faster convergence to equilibrium, (2) a more robust parallel cold-start mechanism even for very large configurations, (3) an option for dynamic load balancing, (4) an option for using double precision for force computation.

We shall discuss two of the most time consuming computational kernels in SOTON_PAR: movout, which performs geometric hashing, and force, which performs the force evaluations.

\subsection{Geometric Hashing}

The link-cell method [4] is commonly used to speed up MD force calculations by minimizing the number of neighboring atoms that must be checked for possible interaction (based on a cut-off distance of $r_{c}$, beyond which particle interaction is assumed to be negligible). At each time step, all the atoms are hashed into 
three-dimensional bins or cells of side length at least $r_{c}$. Each particle can thus interact only with atoms within the same bin or the 26 surrounding bins. Hashing the atoms can be easily parallelized and requires only $O(N)$ work. Only 13 of

the neighboring bins need to be examined if Newton's Third Law $\left(f_{i j}=-f_{j i}\right)$ is applied. The link-cell method is memory efficient, requiring only $O\left(N_{b}^{3}\right)$ storage for the 3 -D bins, 9 real vectors of length $N\left(x, y, z, v_{x}, v_{y}, v_{z}, f_{x}, f_{y}, f_{z}\right.$ for the positions, velocities and forces, respectively) and an integer vector of length $N$ for storing the linked list.

Another common technique is to construct and maintain for each atom, a list of neighboring atoms [10]. This list is updated every few time steps. The advantage provided by the neighbor list is that once the list is built, examining it for possible interaction is much quicker than checking all other atoms in the neighboring bins. However, the neighbor list for each atom commonly grows quite long (70 or more atoms per list), and the resulting high cost in memory is prohibitive for very large scale MD calculations.

SOTON_PAR is based on a spatial decomposition and uses the link-cell method. Each processor is responsible for a spatial region and the corresponding subset of 3-D bins. SOTON_PAR uses message passing code to exchange atoms with neighbor processors as atoms migrate across processor boundaries. A highly nonuniform distribution of particles may result in a serious load-imbalance.

\subsection{DOLIB implementation of movout}

The link-cell algorithm in SOTON_PAR constructs a linked list of atoms for each bin. We implement the link-cell method by performing a reordering or renumbering so that all atoms in a bin are contiguously numbered, e.g., if each bin has 10 atoms, then after the reordering, atoms 1 to 10 are contained in the first bin, atoms 11 to 20 are contained in the second bin, etc. The reordering permits the use of the most efficient DOLIB contiguous block gather/scatter operations on long vectors.

Two passes are required for the geometric hashing and reordering. The first 
pass performs the geometric hashing and stores the result of the mapping. For each bin, we compute the number of atoms to be assigned. We then set up and allocate storage for each bin using a pointer array $x l$ ist. The second pass then performs the actual reordering and data movement. Note that vectors $\left(f_{x}, f_{y}, f_{z}\right)$ are used as temporary storage and will be cleared again for force computation.

The geometric hashing and reordering is performed in several routines:

adjustxyz: After a time step, particles close to the boundary might exit the canonical box $[-0.5,0.5] \times[-0.5,0.5] \times[-0.5,0.5]$. We implement a periodic boundary condition by adjusting the $(x, y, z)$ coordinate to reintroduce the particle at the opposite face. Hence,

$$
\begin{aligned}
& \text { if } \quad(x>0.5) \quad x=x-1.0 \\
& \text { if } \quad(x<-0.5) \quad x=x+1.0
\end{aligned}
$$

hashxyz: Geometric hashing is performed as a simple divide and integer truncation operation. An atom with coordinates $(x, y, z)$ will be hashed to $3-\mathrm{D}$ bin $(i, j, k)$ by

$$
\begin{aligned}
i & =1+\operatorname{int}\left((x-(-0.5)) / r_{c}\right) \\
j & =1+\operatorname{int}\left((y-(-0.5)) / r_{c}\right) \\
k & =1+\operatorname{int}\left((z-(-0.5)) / r_{c}\right)
\end{aligned}
$$

The result of the hashing is stored in array imap for use in the second pass. The array imap requires $N_{b}^{3}$ words of memory.

setxlist: We use a pointer array $x l$ ist to mark for each bin, the beginning position (or label) of each contiguous block of atoms assigned to the bin.

Let $m_{i}$ be the number of particles assigned to the $i^{\text {th }}$ bin, then $x$ list $(1)=1$, $x \operatorname{list}(2)=1+m_{1}, x \operatorname{list}(3)=x \operatorname{list}(2)+m_{2}, \cdots, x \operatorname{list}(k+1)=x \operatorname{list}(k)+m_{k}$, etc. The array xlist requires $N_{b}^{3}$ words of memory. 
setuplist: We shall use $\mathrm{xl}$ ist as pointer to the next available storage position for each bin. Thus as we assign particles into bins, $x l i s t$ has to be incremented in parallel with an atomic operation. We avoid the use of inefficient lock/unlock by using the DOLIB axpbyz operation.

copyxyz: Once the assignment mapping is determined, vectors $(x, y, z)$ and $\left(v_{x}, v_{y}, v_{z}\right)$ are permuted using $\left(f_{x}, f_{y}, f_{z}\right)$ as temporary storage. Vectors $\left(f_{x}, f_{y}, f_{z}\right)$ are then cleared again before the force computation. The arrays $(x, y, z),\left(v_{x}, v_{y}, v_{z}\right)$ and $\left(f_{x}, f_{y}, f_{z}\right)$ require $9 N$ words of memory for $N$ atoms. If the forces $\left(f_{x}, f_{y}, f_{z}\right)$ are to be computed in double precision, $12 N$ words are required.

adjustxlist: Finally we reset $\mathrm{xlist}$ to point to the beginning position of each contiguous block.

Note that as the MD system converges to an equilibrium state, most of the particles will be hashed into the same bins to which they belonged in the previous time step, thus requiring only a small amount of data movement.

In Section 3 we list the proportion of time spent in each routine.

\subsection{Force evaluation}

We take advantage of Newton's 3rd Law $\left(f_{i j}=-f_{j i}\right)$ to compute a force interaction only once for each atom pair. This means only 13 (instead of 26) neighboring bins must be examined. Lomdahl [5] describes the selection of 13 neighbors as a particular "3D interactions path", though in fact any stencil of 13 neighboring bins can be chosen, if consistently used.

For simplicity, we consider a two-dimensional partition of the computation. Thus, we group all force interactions for each column of $N_{b}$ cells of the $3 \mathrm{D}$ mesh of bins as one unit. The $N_{b} \times N_{b}$ columns are then block partitioned and assigned to individual processors. Because all atoms in each bin are contiguously ordered (see Section 2.2), DOLIB's efficient contiguous block gather/scatters can be used. Moreover, we exploit data reuse by selecting the 13 neighboring cells to be 

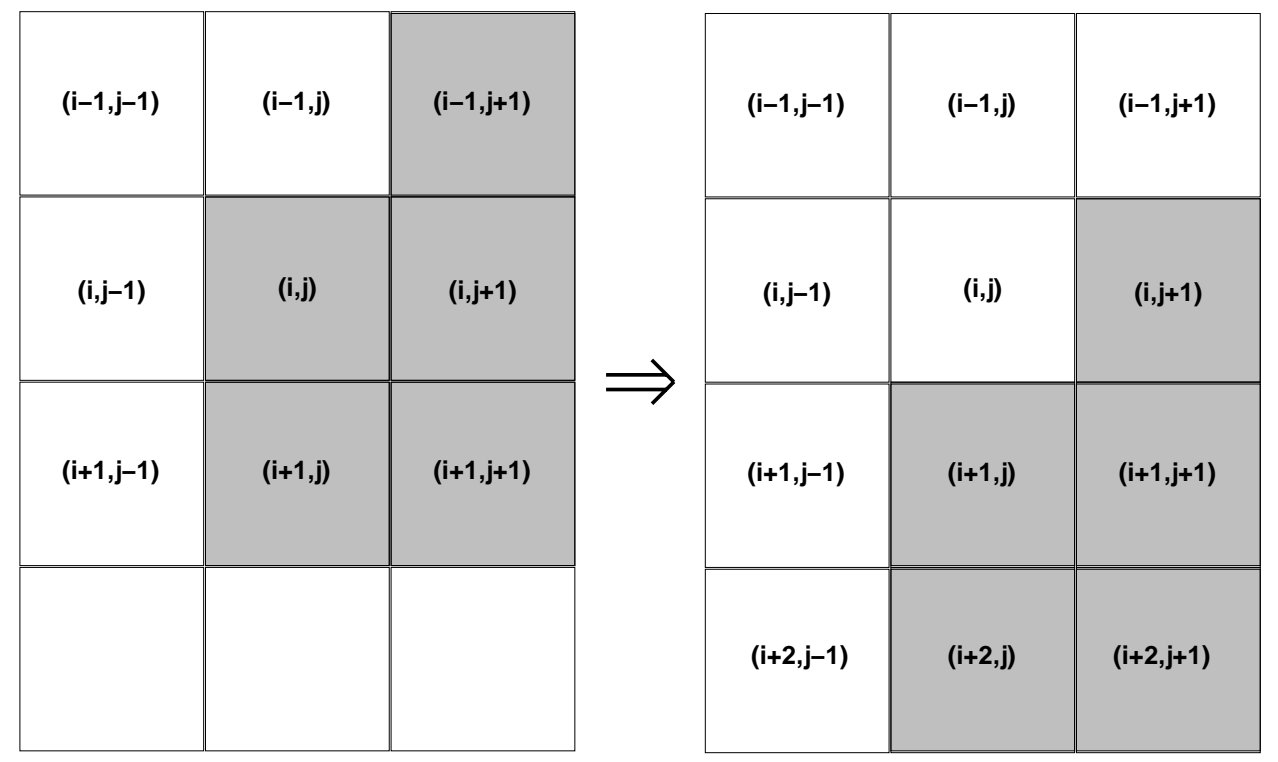

Figure 2.1: Top view of columns used in processing column $(i, j)$. Only two new columns are needed in processing column $(i+1, j)$.

in 5 neighboring columns (see Figure 2.1). To process column $(i, j)$ we require data from neighboring columns $(i-1, j+1),(i, j+1),(i+1, j),(i+1, j+1)$; to process the next column $(i+1, j)$, data in columns $(i+1, j),(i, j+1),(i+1, j+1)$ can be reused. Only data for two new columns $(i+2, j)$ and $(i+2, j+1)$ must be brought in. This organization reduces the amount of communication required for gather/scatter operations.

Another advantage of this reorganization is that force evaluation in each of the bins requires no further communication. Automatic thread parallelization by the Intel MP node Paragon compiler is thus simplified.

\subsection{Dynamic Load Balancing}

We include an option to estimate a work measure for each column, using this to distribute columns to processors to avoid load imbalance. One simple measure of work load is the total number of atoms in the column. For a uniform distribution of atoms in the domain, this is generally sufficient to attain good load-balance. However, for nonuniform distributions, a more reasonable work measure for each 
bin is to count the total number of possible atom-atom interactions among other particles in the 13 neighboring bins. Both of these work estimates are provided as options to the load-balancing routine.

The load balancing strategy then computes the overall total and average amount of work for each processor, assigning columns to processors to satisfy this average using a greedy algorithm. Future versions will provide a more sophisticated bin-packing algorithm. However, for the simple Lennard-Jones fluid simulations, each bin has approximately the same number of atoms and induces almost no load imbalance.

\subsection{Shifted Force Potential}

SOTON_PAR implements a shifted force Lennard-Jones 6-12 potential [1, page 145] where a small linear term is added to the potential so that it is continuous and its derivative is zero at the cutoff distance. The code also computes the virial and potential energy along with force calculations. Each atom-pair takes 8 flops and 1 compare to determine whether they are within the cut-off distance. If they are, then another 29 flops, 2 divides and 1 square root operation are further required. In our experience, the accumulation into total potential energy may encounter catastrophic cancellation that can yield as few as 4 significant digits of precision. We have implemented an option using separate double precision variables $v_{\text {pos }}$ and $v_{n e g}$ for accumulating positive and negative potential values, which reduces the amount of numerical cancellation and produces more consistent results as the number of processors is varied.

\section{Parallel Performance}

Our parallel codes were tested on an Intel MP node Paragon system. Each MP node contains 3 CPUs and at least 64MBytes of memory in a local shared memory configuration. By default, one CPU is a dedicated message co-processor. The second CPU runs the main computational thread. The third CPU can be utilized by 
automatic thread parallelization by the Paragon MP Fortran compiler. The MP node can also be configured in "turbo" mode where all three CPUs are dedicated for computation but with some degradation in communication performance. All our MD computations were done in single precision in non-turbo mode, although we have also implemented a double precision option for force computations.

We tested our code with a benchmark problem described in Plimpton [8, page 23] for a Lennard-Jones 6-12 potential with reduced density $\rho=0.8442$, and reduced temperature $T=0.72$. The system is initialized with a fcc lattice and randomized velocities chosen from a Boltzmann distribution. The integration time step is 0.00462 in reduced units, and cut-off distance is $r_{c}=2.5 \sigma$. Plimpton estimates there are about 55 neighbors interacting with each atom at every time step (out of about 177 atom-pairs examined). If we count a divide as 5 flops and a square root also as 5 flops, then (from Section 2.5) each atom requires approximately $9 * 177+44 * 55=4013$ flops for force evaluation. Updating the velocities, positions and accumulation of total kinetic energy in movea and moveb requires another 30 flops per atom.

Table 3.1 shows averaged run times per time step for a 500,000 atom simulation $(50 \times 50 \times 50$ lattice $)$. Initialization and setup time are excluded. We achieved faster run times without the overhead of computing work measure and dynamic load balancing, since the problem is already well-balanced. The serial run was performed using the same "parallel" code on a single processor. Note that no messages were generated in the DOLIB gather/scatter since on a single processor these are translated into memory copies.

For the 500,000 atom problem, our code achieved a speed of $0.175 \mathrm{~ms} /$ atom per time step on a single processor (with parallel threads) with no messages generated. About $75 \%$ of the overall time (per time step) was spent in force computation and about $20 \%$ in performing reordering in movout. Within movout, about $42 \%$ of time was spent in performing 3-D bin hashing (first pass) in hashxyz, about $17 \%$ in allocating storage with DOLIB axpbyz operation in routine setuplist and about $35 \%$ in performing (second pass) actual reordering of vectors $(x, y, z$, 
$\left.v_{x}, v_{y}, v_{z}\right)$ in routine copyxyz. On 64 processors with parallel threads, the speed decreased to about $0.31 \mathrm{~ms} /$ atom per processor per time step. About $60 \%$ was in force and $30 \%$ in movout. Within movout, about $27 \%$ was in hashxyz, $21 \%$ in setuplist and about $38 \%$ was in copyxyz. Note however, there is message passing and servicing of remote memory requests even within the force computations.

By comparison, the original SOTON_PAR host/node codes required about 17.1 sec on 8 processors for a 256,000 atom $(40 \times 40 \times 40$ lattice $)$ with no parallel threads. This is about $0.534 \mathrm{~ms} /$ atom/processor. Our code (with no parallel threads) takes about $10.4 \mathrm{sec}$ per time step. This is about $0.333 \mathrm{~ms}$ /atom. With parallel threads enabled, the average time reduced to $7.7 \mathrm{sec}$ per step, or $0.25 \mathrm{~ms} /$ atom/processor.

On a much larger problem with 32 million atoms $(200 \times 200 \times 200$ lattice $)$ our code takes about $205.3 \mathrm{sec}$ on 32 processors and $103.7 \mathrm{sec}$ on 64 processors, which is about $0.207 \mathrm{~ms} /$ atom per processor. This yields an approximate overall rate (including message passing overhead) of $19.5 \mathrm{MFlop} / \mathrm{sec}$ for each node (with parallel threading enabled). Although we used single precision in our codes, our run times compare quite favorably with the times of $0.24 \mathrm{~ms} /$ atom/processor on the Intel Delta [8] and $0.26 \mathrm{~ms} /$ atom/processor [2] (performed in double precision) on a 1024 processor CM- $5^{2}$ both using a spatial decomposition and linked-cell method.

\section{Acknowledgments}

The authors take this opportunity to express appreciation to Bob Marr, Ron Peierls and Joe Pasciak for the IPX package, which simplified the development of DOLIB. We also thank Bill Shelton and David Walker for their insight and advice on Molecular Dynamics Simulations.

\footnotetext{
${ }^{2}$ The code used on the CM-5 computes about 7,000 flops per atom.
} 
Table 3.1: Averaged runtimes (in sec) per timestep for 500,000 nodes problem.

\begin{tabular}{|r|r|r|}
\hline \hline Processor & 1 thread & 2 threads \\
\hline \hline 1 & 137.5 & 95.4 \\
2 & 73.1 & 51.8 \\
4 & 37.6 & 27.2 \\
8 & 19.7 & 13.2 \\
16 & 10.3 & 7.1 \\
32 & 5.6 & 4.3 \\
64 & 3.3 & 2.4 \\
\hline
\end{tabular}




\section{Appendix}

In this appendix, we list the RATFOR source code for some of the key routines used in movout permutation and force computations.

\section{1. subroutine movout}

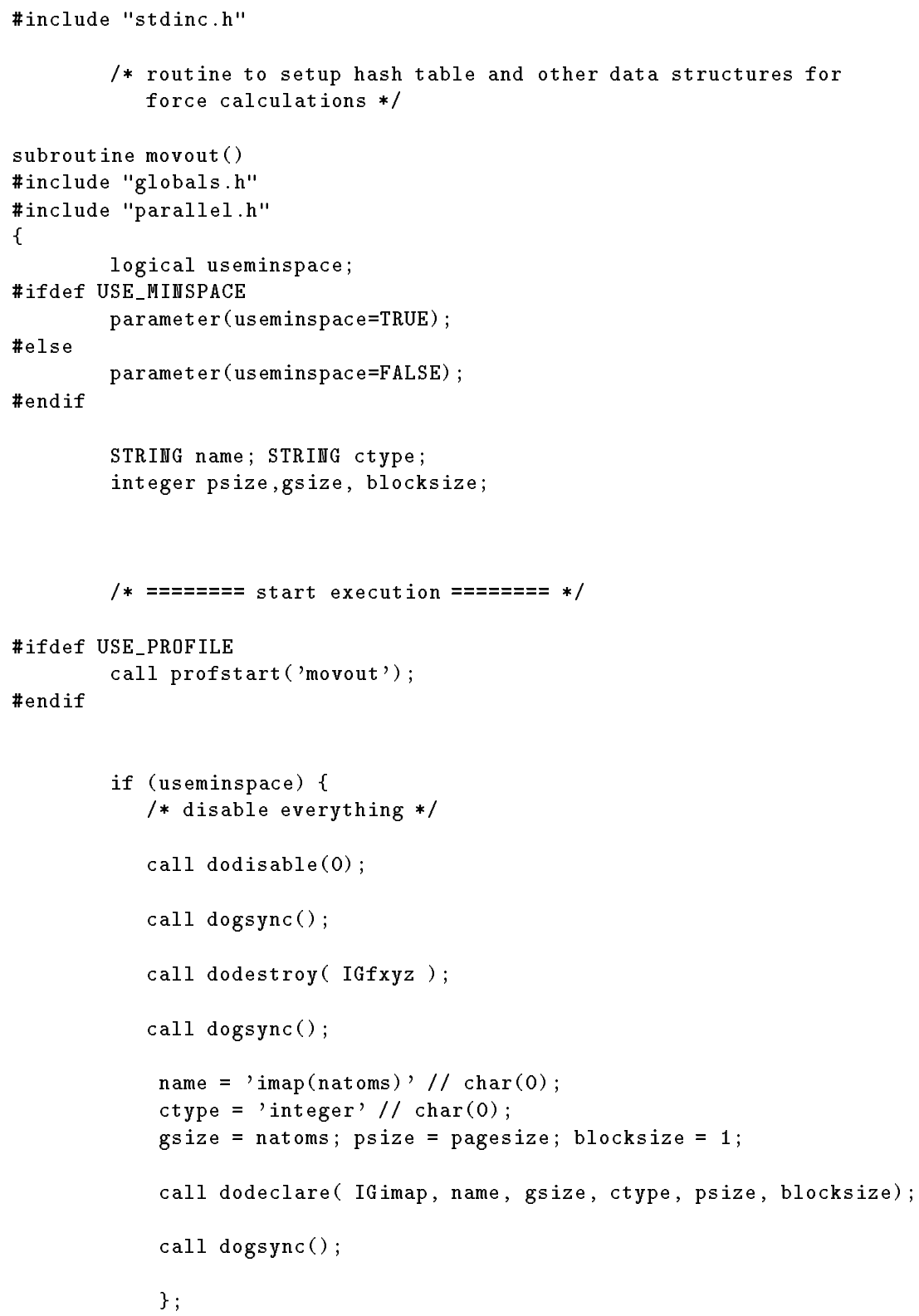




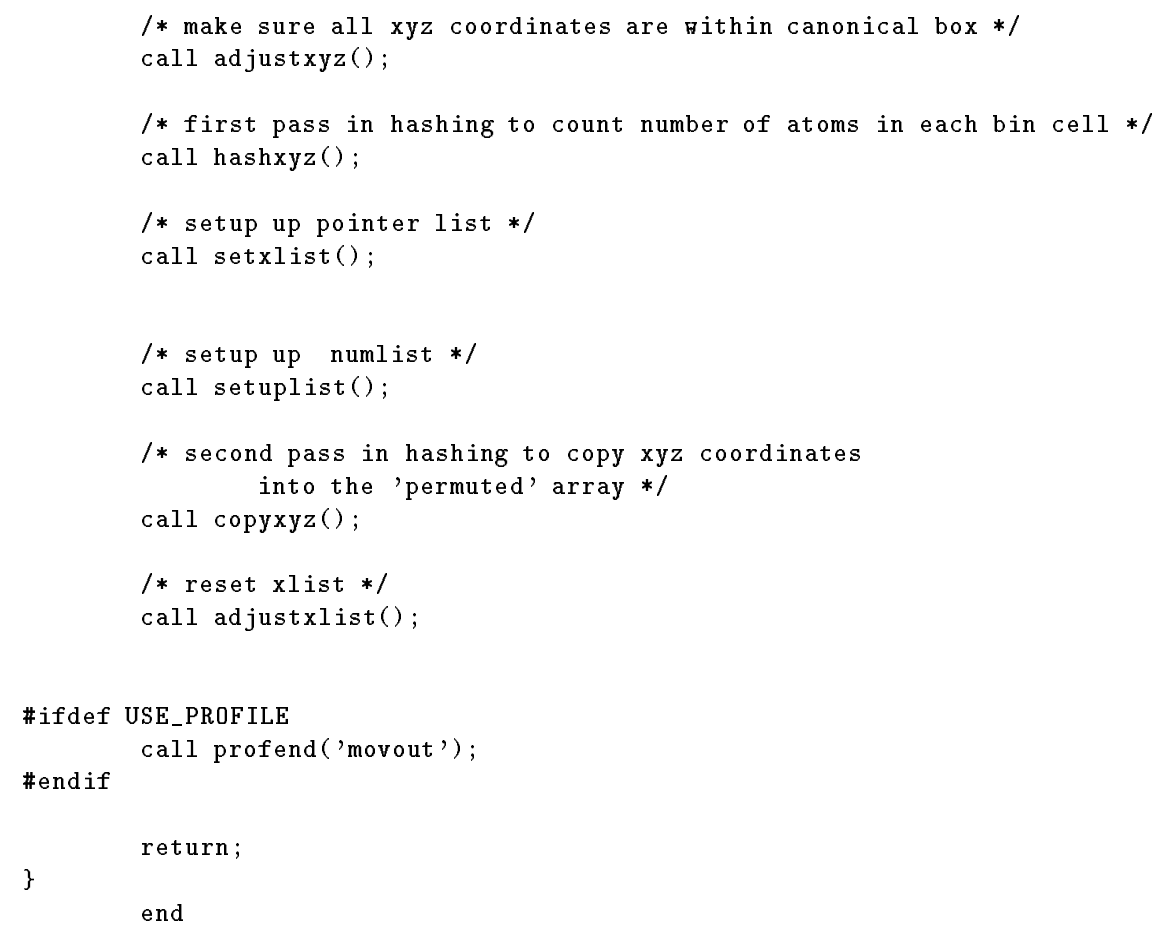

\section{2. subroutine adjustxyz}

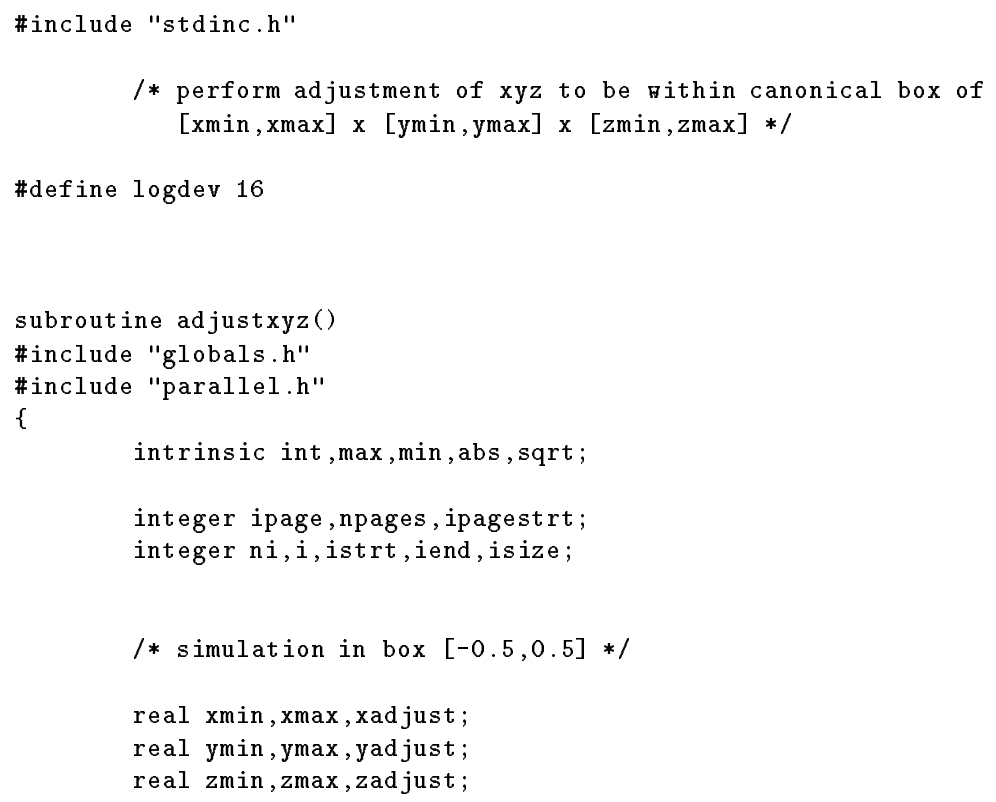


parameter $(x \operatorname{xin}=-0.5, x \max =0.5, \operatorname{xadjust}=(x \max -x \min ))$; parameter $(y \min =-0.5, y \max =0.5$, yad just $=(\operatorname{ymax}-y m i n))$; parameter $(\operatorname{zmin}=-0.5, z \max =0.5, \operatorname{zadjust}=(\operatorname{zmax}-z \min ))$;

real one,zero;

parameter $($ one $=1.0$, zero $=0.0)$;

real xyz(3,pagesize);

\#define $r x(i) \operatorname{xyz}(1, i)$

\#define ry(i) $x y z(2, i)$

\#define $r z(i)$ xyz $(3, i)$

logical isxok, isyok, iszok, isallok, isformatted;

$/ *=======$ start execution $========* /$

\#ifdef USE_PROFILE

call profstart('adjustxyz');

\#endif

call dogsync();

npages $=$ int $($ natoms $/$ pagesize $)+1 ;$

ipagestrt=myid ;

call dodisable( IGxyz );

/* scatter operation */

call openrbuf( IGxyz, one, zero);

doloop4(ipage, ipagestrt, npages, nproc) \{

istrt $=1+$ ipage*pagesize;

iend $=\min ($ natoms, istrt+pagesize-1);

isize $=$ iend - istrt +1 ;

if (isize $<0$ ) $\{$ break; ;

call dowait(dobrgather( IGxyz, 3*isize, idxxyz(1,istrt), xyz));

if (IDEBUG >= 3 ) \{

rrite(logdev,*) 'adjustxyz: xyz before update '; rrite(logdev, 9001) (istrt+(i-1), rx(i), ry(i), rz(i), i=1,isize);

9001 format ((1x,i6,' :',3(1x,1pe14.5)));

\} ;

doloop (i,1,isize) \{

isallok = TRUE;

if $(r x(i)<x m i n)\{r x(i)=r x(i)+x a d j u s t ;$ isallok $=$ FALSE; $\}$ if $(r x(i)>\operatorname{xmax})\{r x(i)=r x(i)-x$ adjust; isallok $=$ FALSE; $\}$;

if $(r y(i)<y m i n)\{r y(i)=r y(i)+$ yadjust; isallok $=$ FALSE; $\}$; if $(r y(i)>y \max )\{r y(i)=r y(i)-y a d j u s t ;$ isallok $=$ FALSE; $\}$

if $(r z(i)<z \min )\{r z(i)=r z(i)+$ zadjust; isallok $=$ FALSE; $\}$; if $(r z(i)>z \max )\{r z(i)=r z(i)-$ zadjust; isallok $=$ FALSE; $\}$; 


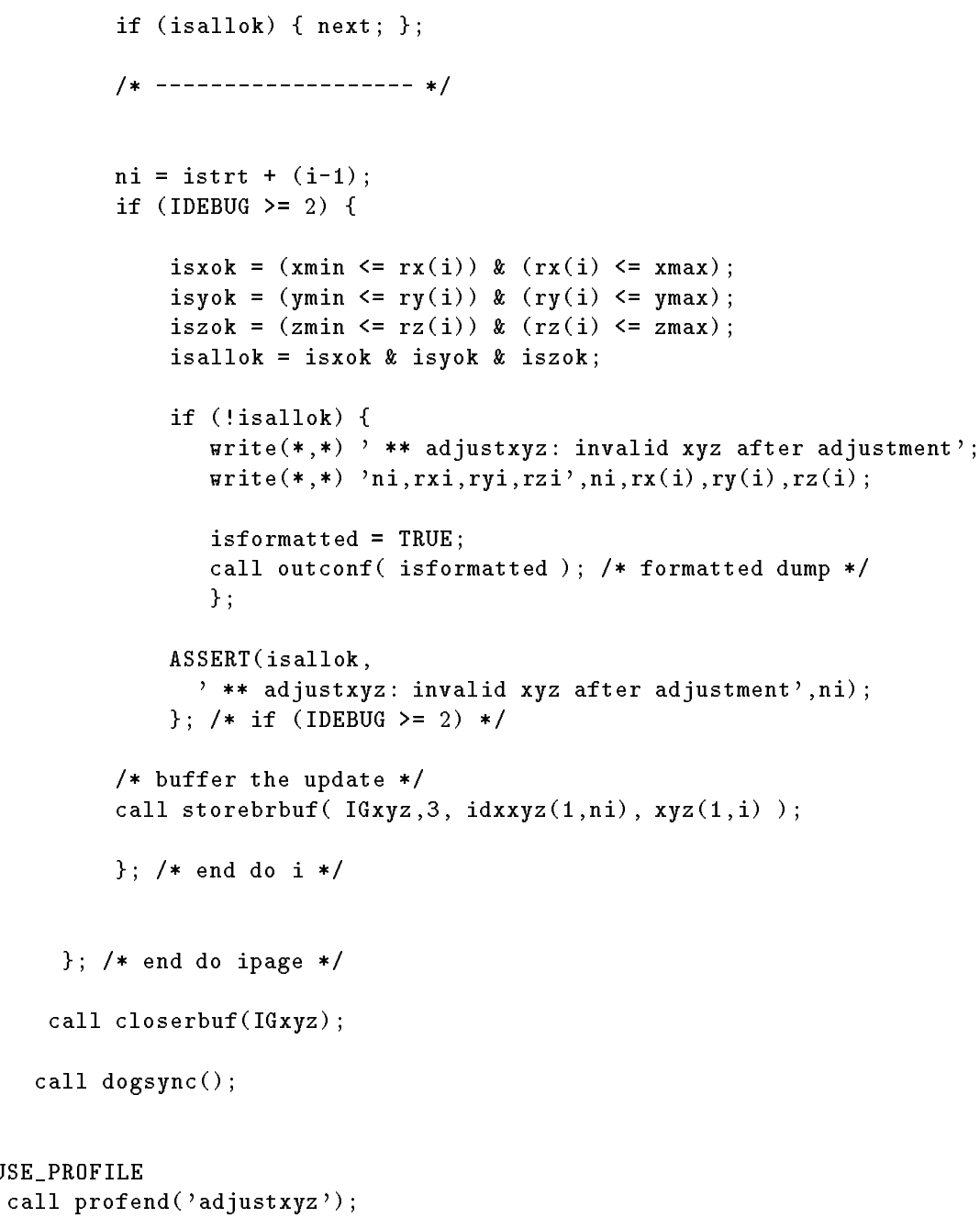

4.3. subroutine hashxyz

\#include "stdinc.h"

/* perform hashing of xyz to count number of atoms in each bin */

\#define USE_WCACHE 1 


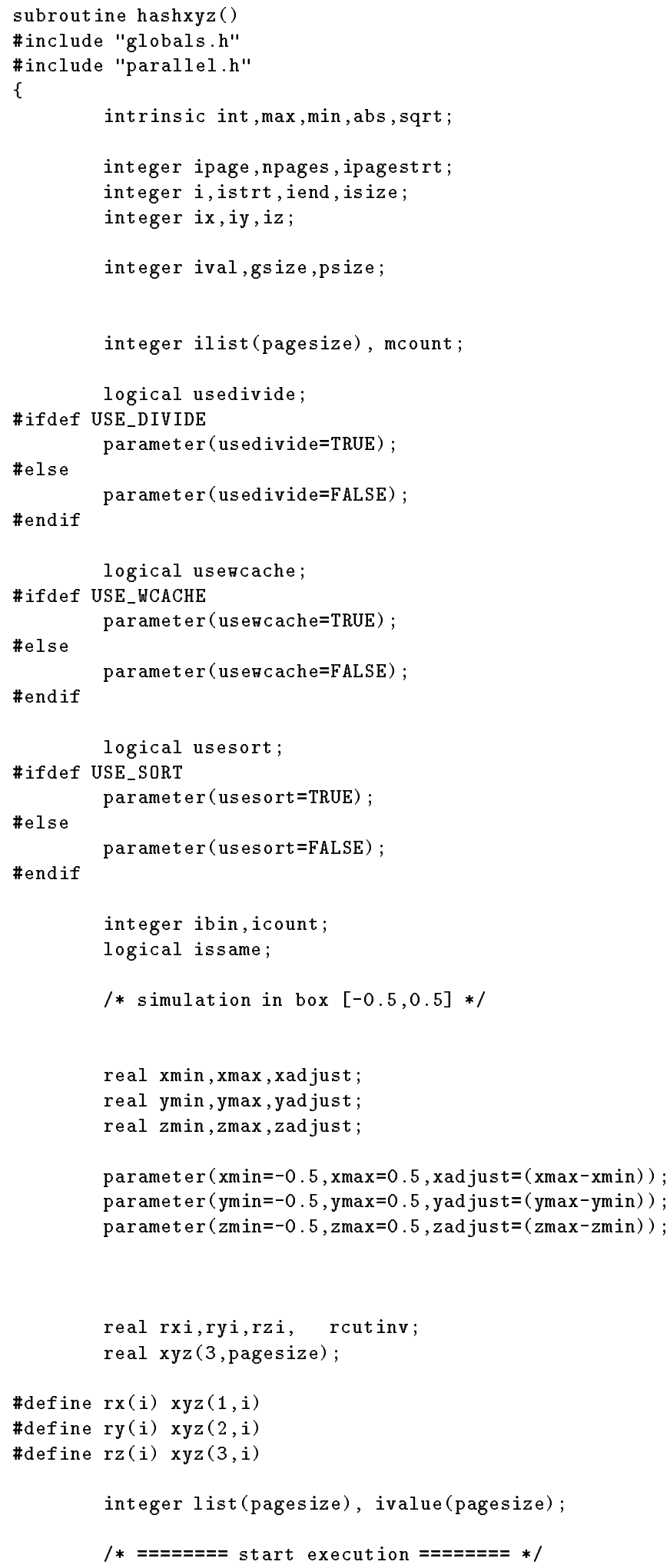


\#ifdef USE_PROFILE
call profstart('hashxyz');

\#endif

call doenable( IGxyz);

call dodisable( IGncount);

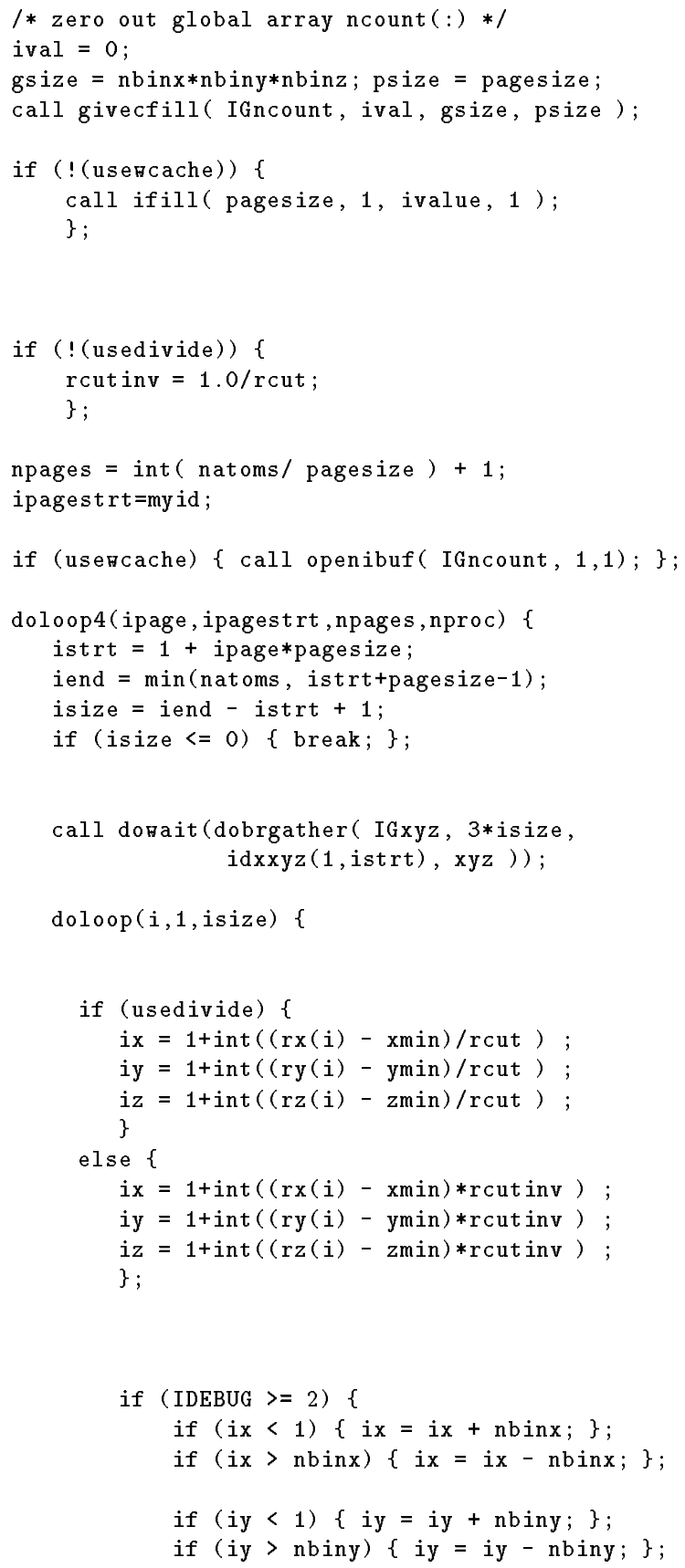




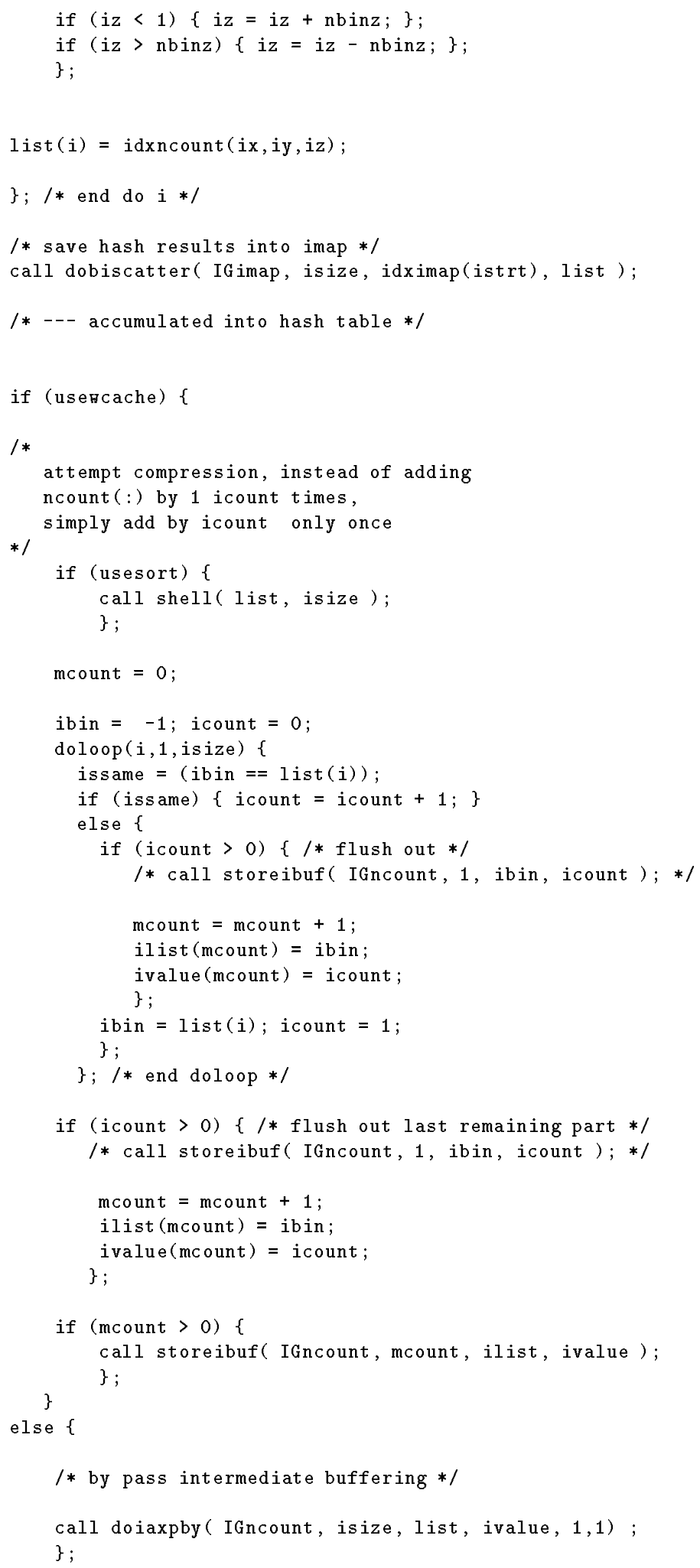




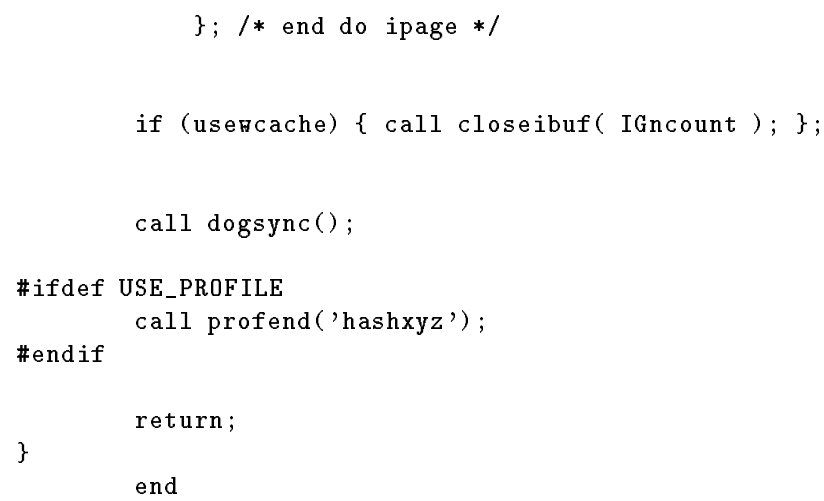

\section{4. subroutine setxlist}

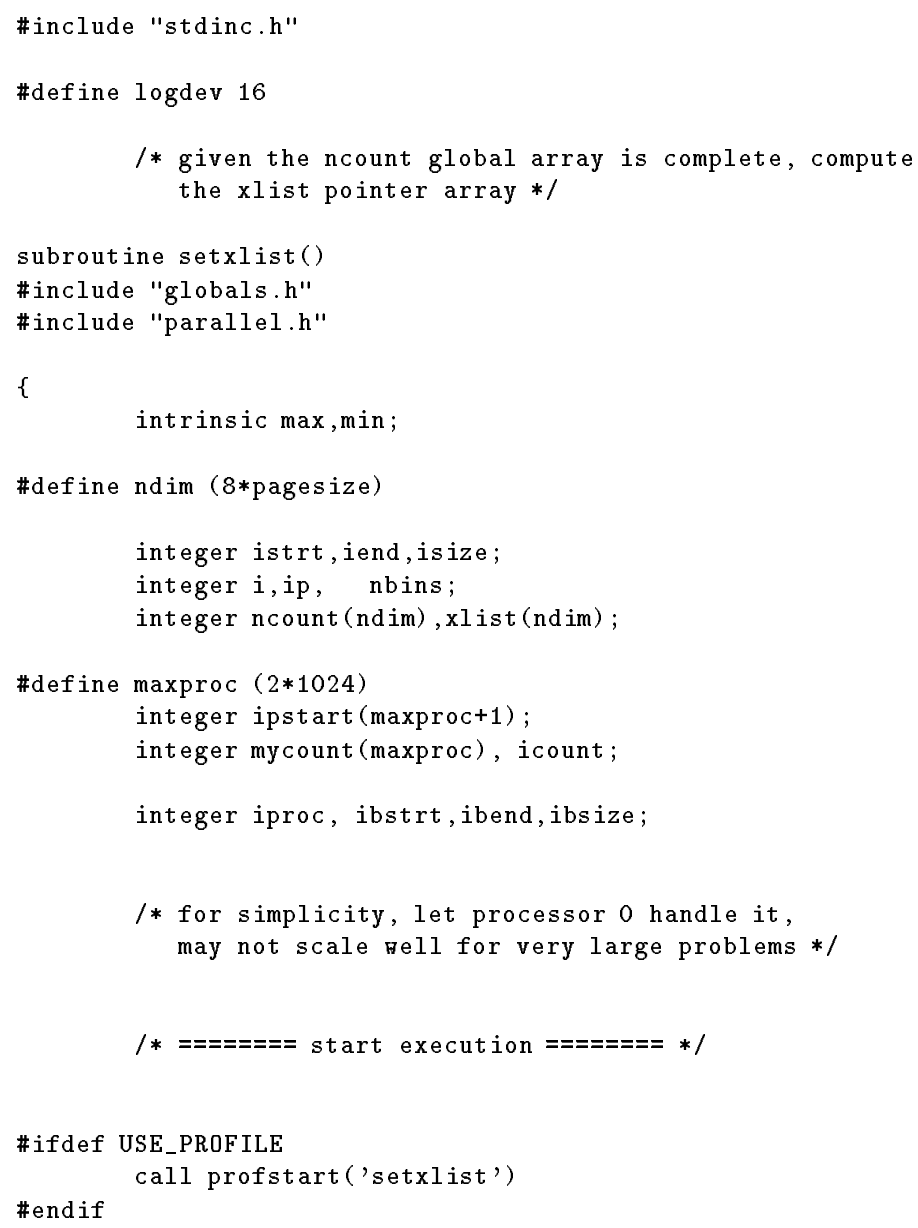




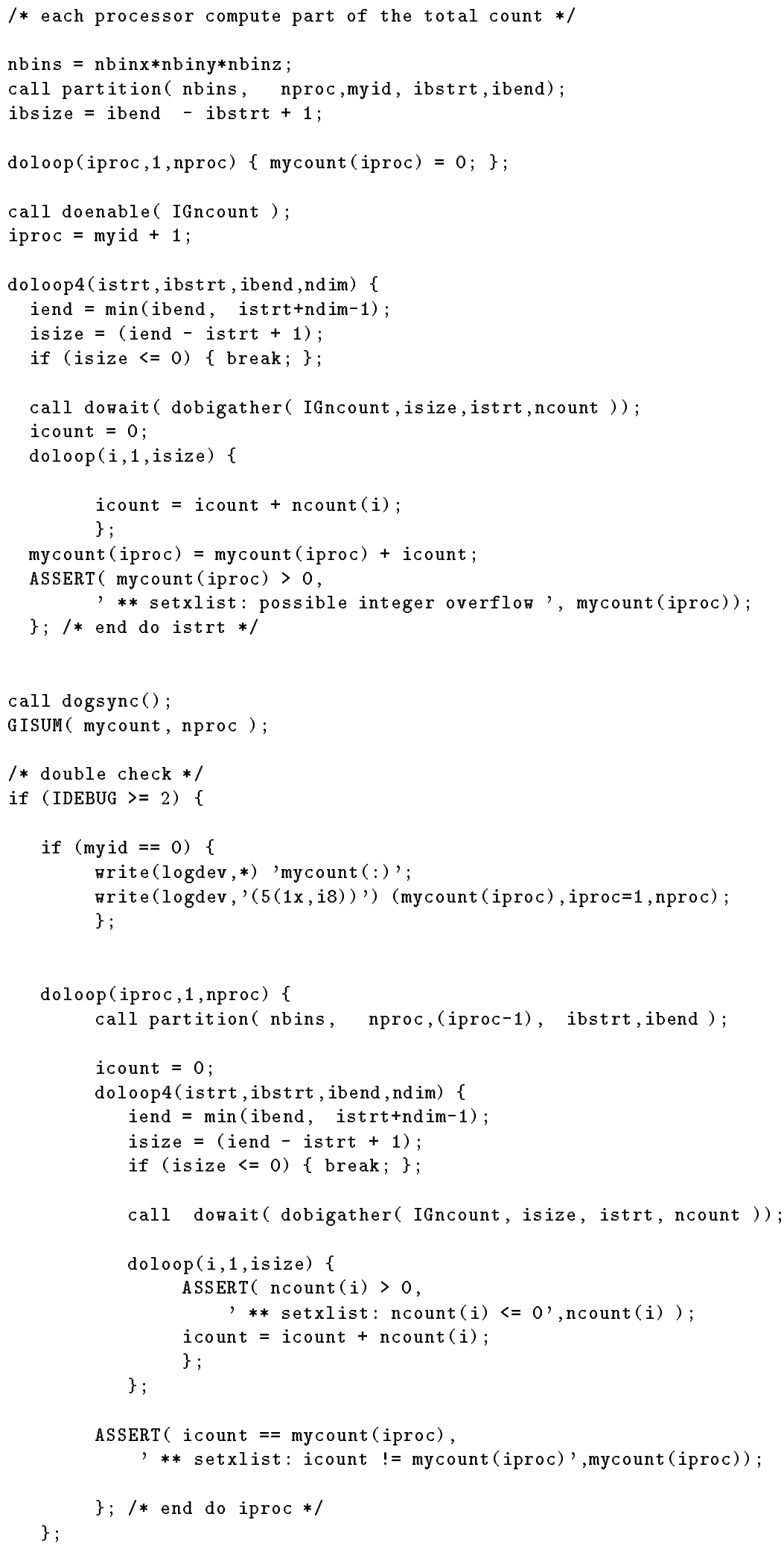




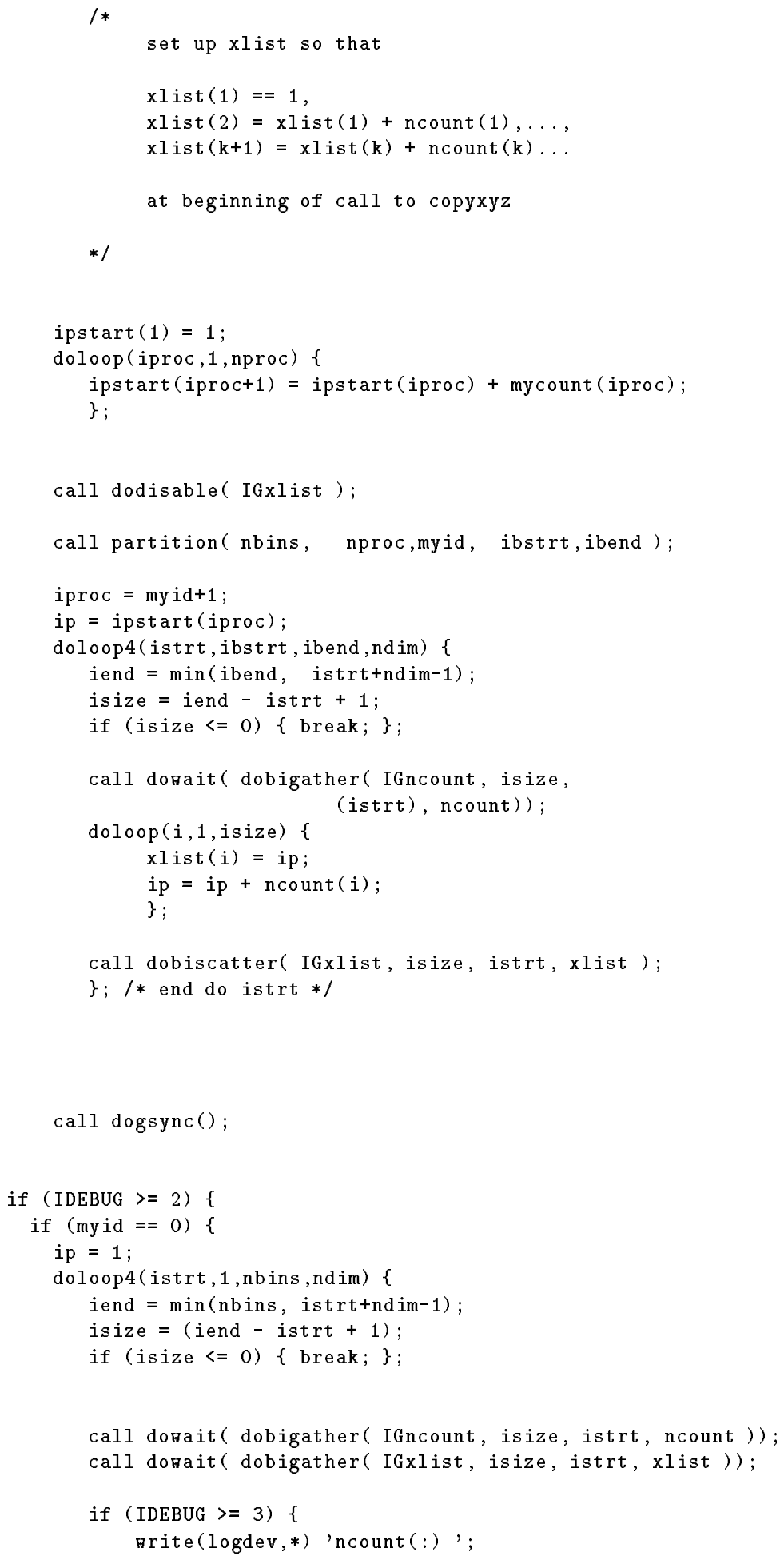




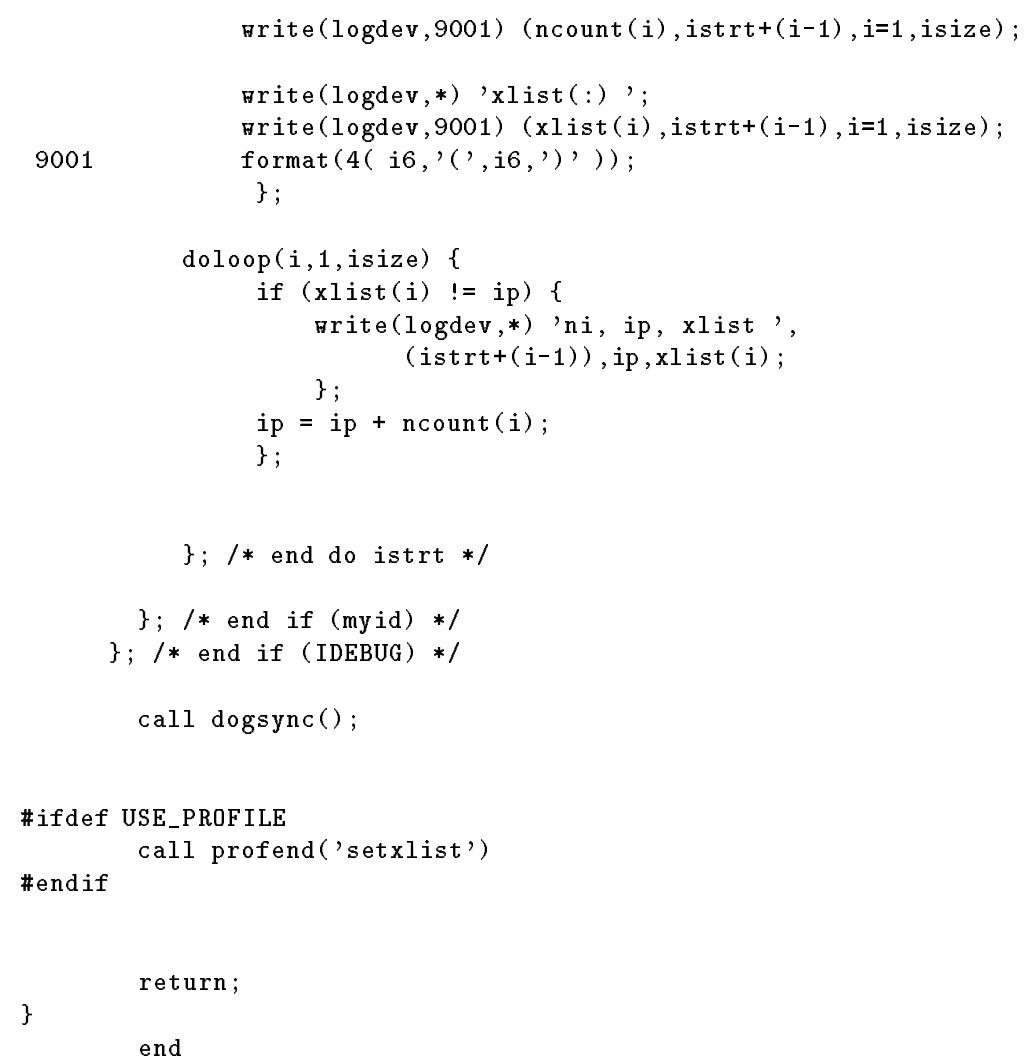

\section{5. subroutine setuplist}

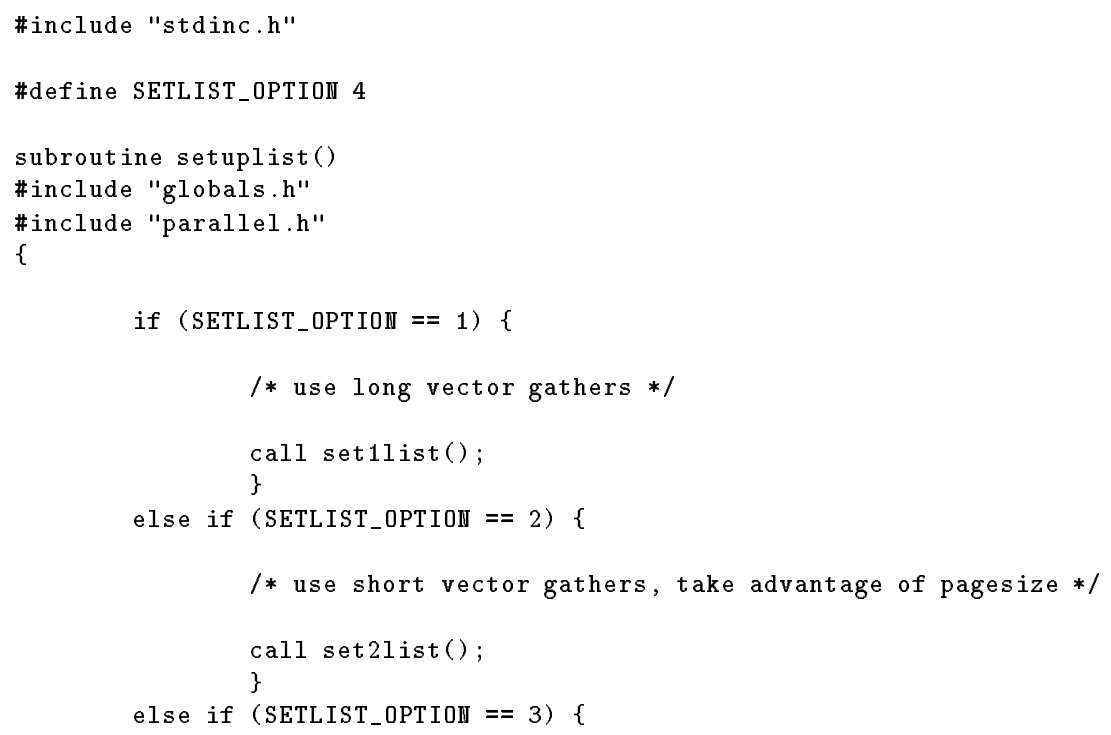




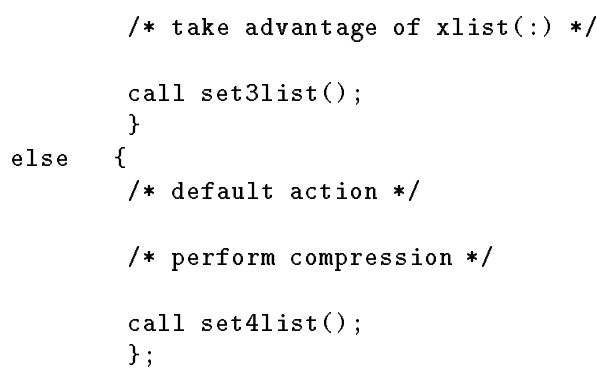

\section{6. subroutine set4list}

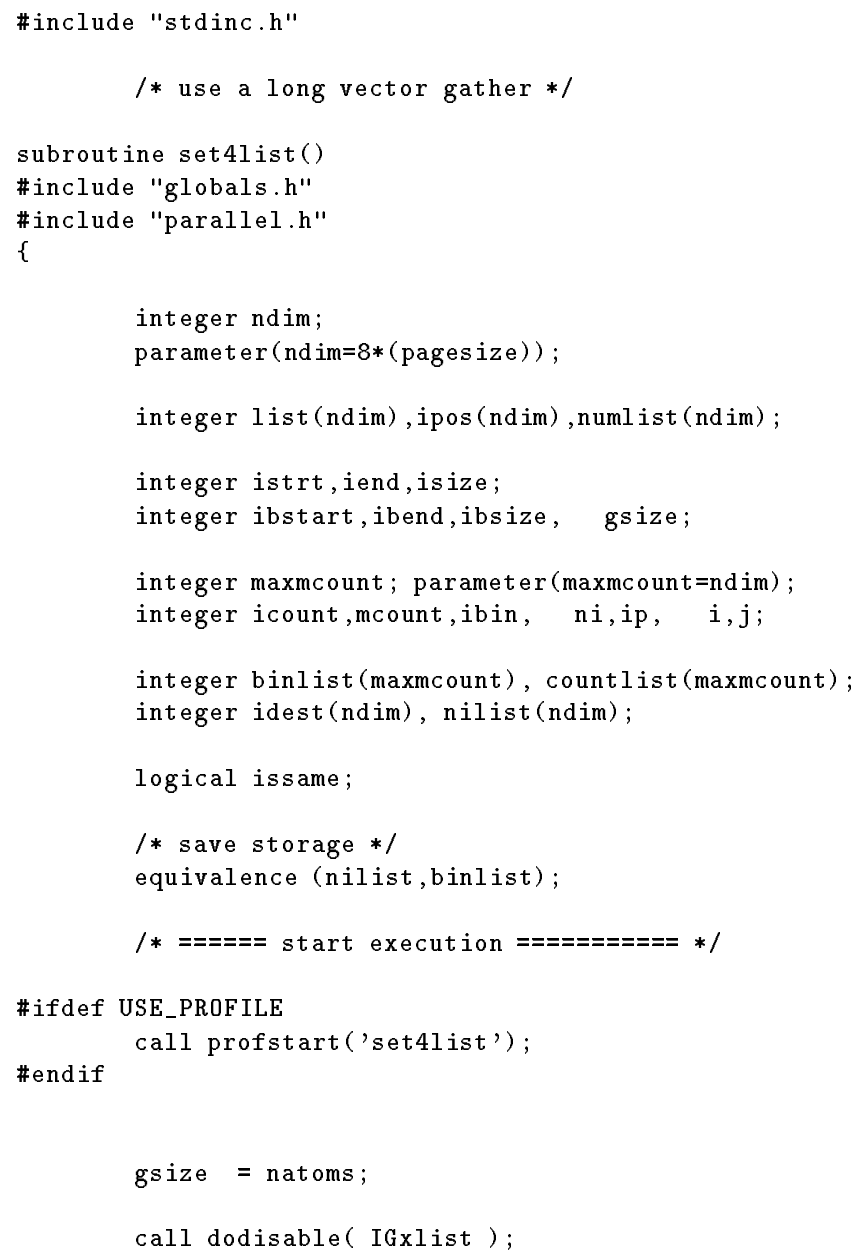




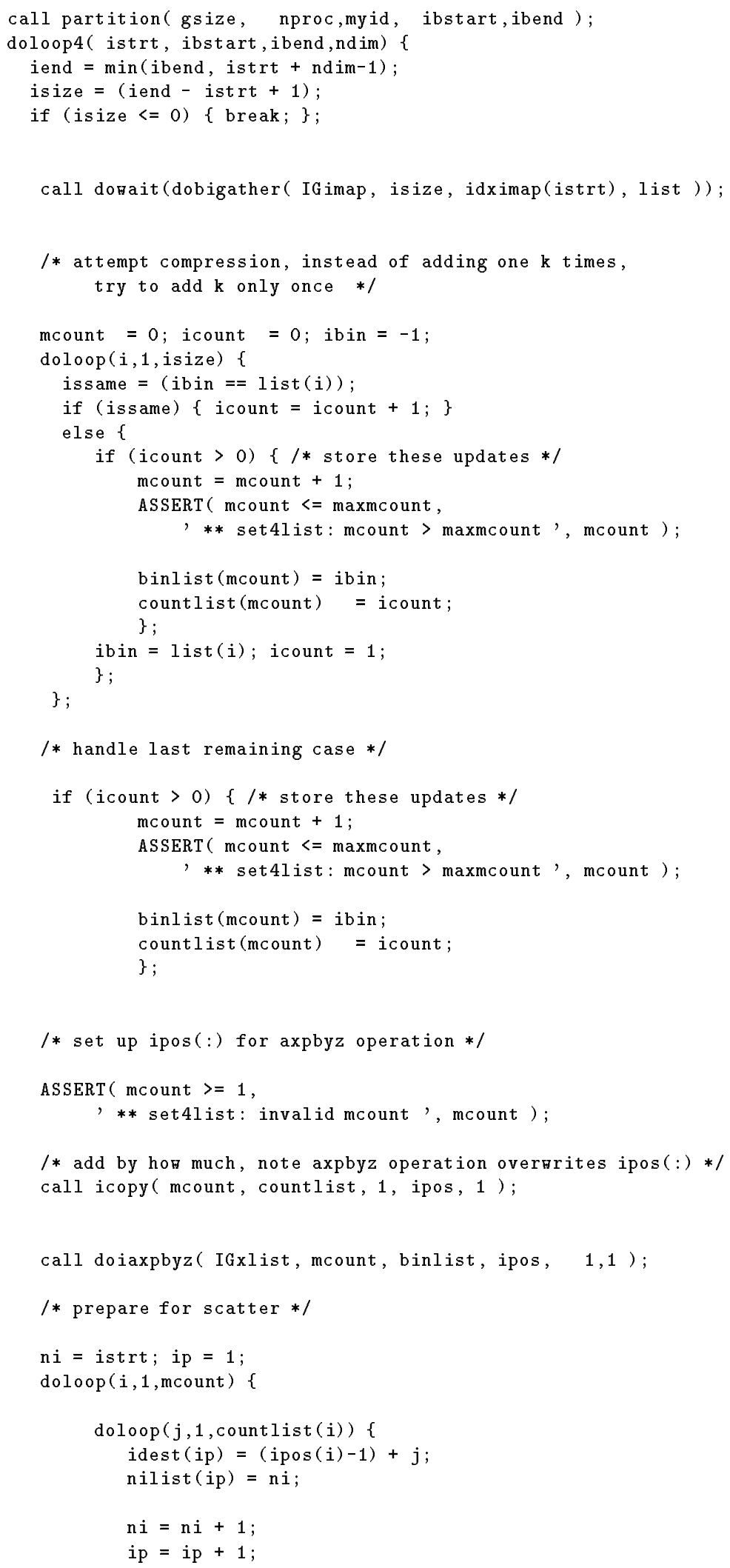




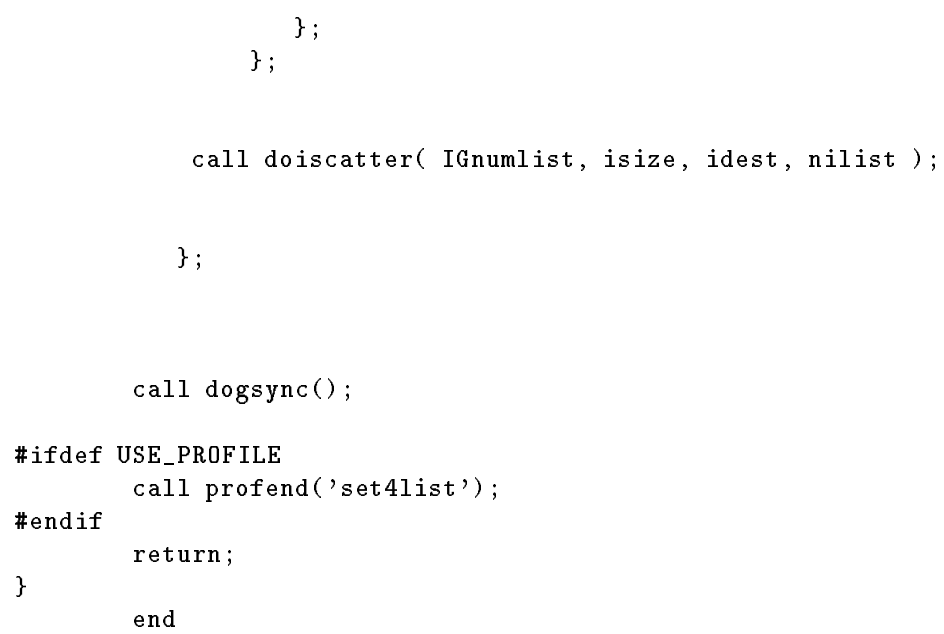

4.7. subroutine copyxyz

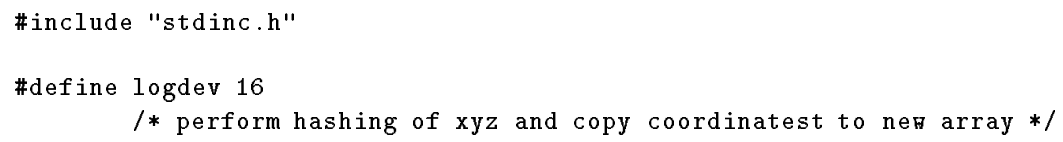


intrinsic int, $\max , \min$, abs, sqrt;

integer ipage,npages, ipagestrt;

integer $i$,istrt,iend,isize, ip,jp, itemp,ni;

integer ix,iy,iz;

/* simulation in box $[-0.5,0.5] * /$

real xmin, xmax, xadjust;

real ymin, ymax, yad just;

real zmin, zmax, zadjust;

parameter $(x \min =-0.5, \operatorname{xmax}=0.5$, xad just $=(x \max -x \min ))$

parameter $(y \min =-0.5, y \max =0.5$, yad just $=(y \max -y \min ))$;

parameter $(\operatorname{zmin}=-0.5, \operatorname{zmax}=0.5, \operatorname{zad} j u s t=(\max -z \min ))$;

logical useminspace;

\#ifdef USE_MIHSPACE

parameter (useminspace=TRUE);

\#else

\#endif

parameter (useminspace=FALSE);

\#define ndim pagesize

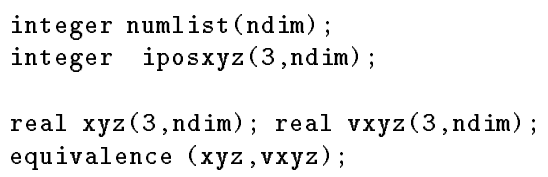

\#define $r x(i)$ xyz $(1, i)$

\#define $r y(i)$ xyz $(2, i)$

\#define $r z(i) \operatorname{xyz}(3, i)$

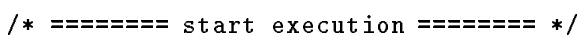

\#ifdef USE_PROFILE

call profstart('copyxyz')

\#endif

/* disable everything */ call dodisable $(0)$;

if (useminspace) \{

/* free storage for imap and recreate fxyz */

/* disable everything, extra safe, and 
encourage memory compaction */

call dodisable (0);

call dogsync();

call dodestroy ( IGimap);

call dogsync();

ctype $=$ 'real' // $\operatorname{char}(0)$;

gsize $=3 *$ natoms; psize $=3 *$ pagesize $;$ blocksize $=1$;

name $=, f x y z(3$, natoms $), / / \operatorname{char}(0)$;

call dodeclare( IGfxyz, name, gsize, ctype, psize, blocksize);

call dogsync();

\};

/* use a "pull" strategy to copy the data */

\#ifdef USE_PROFILE

call profstart('copyxyz:cp xyz');

\#endif

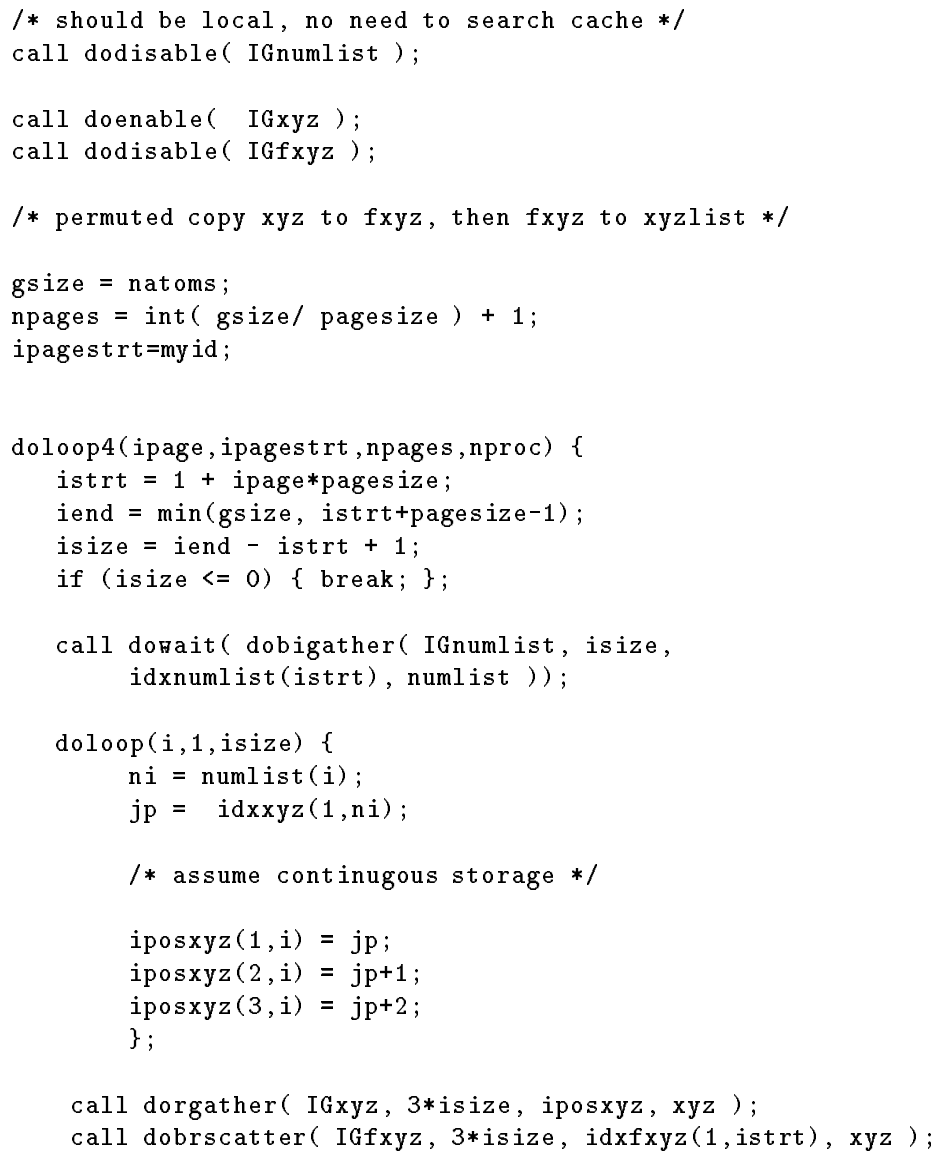




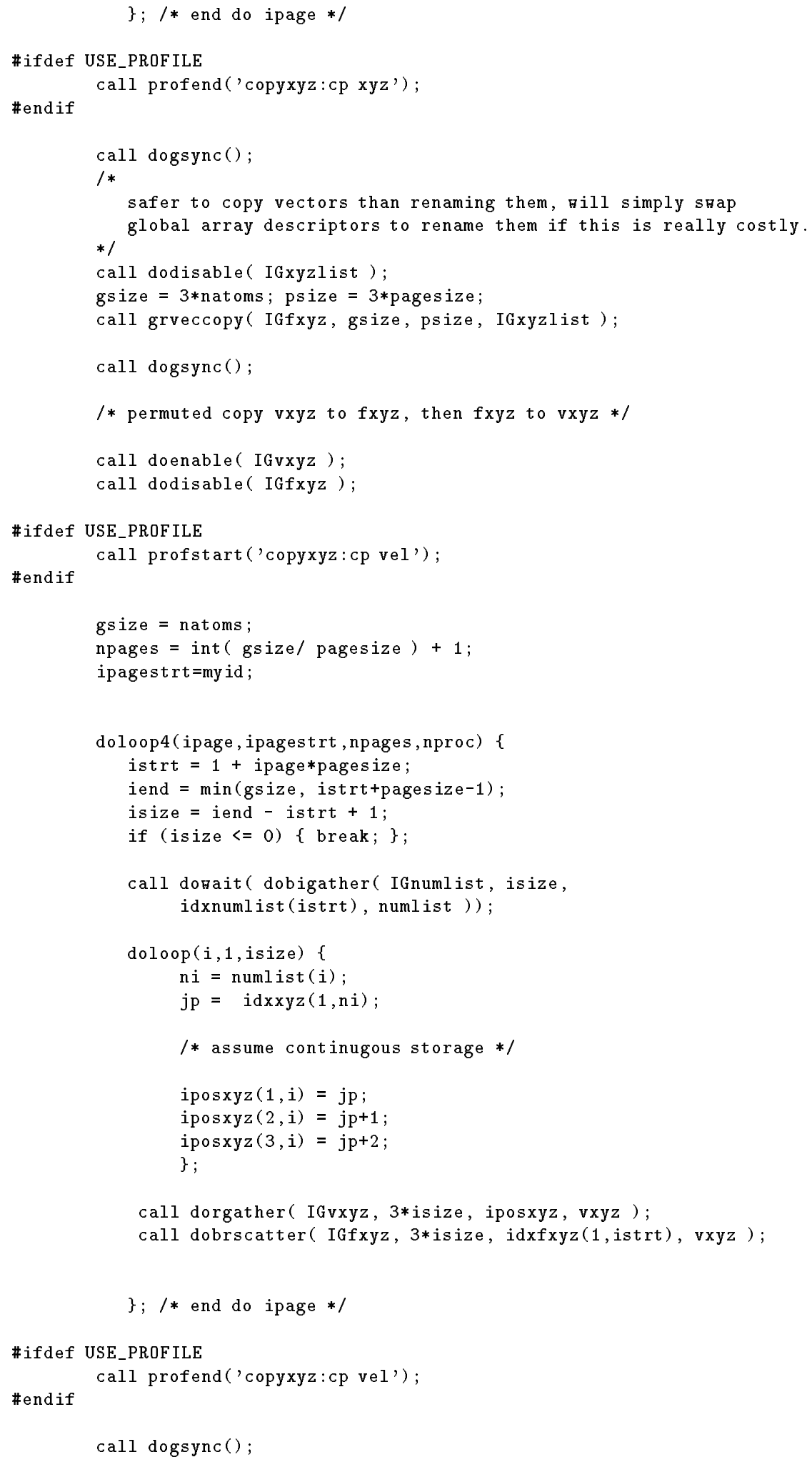




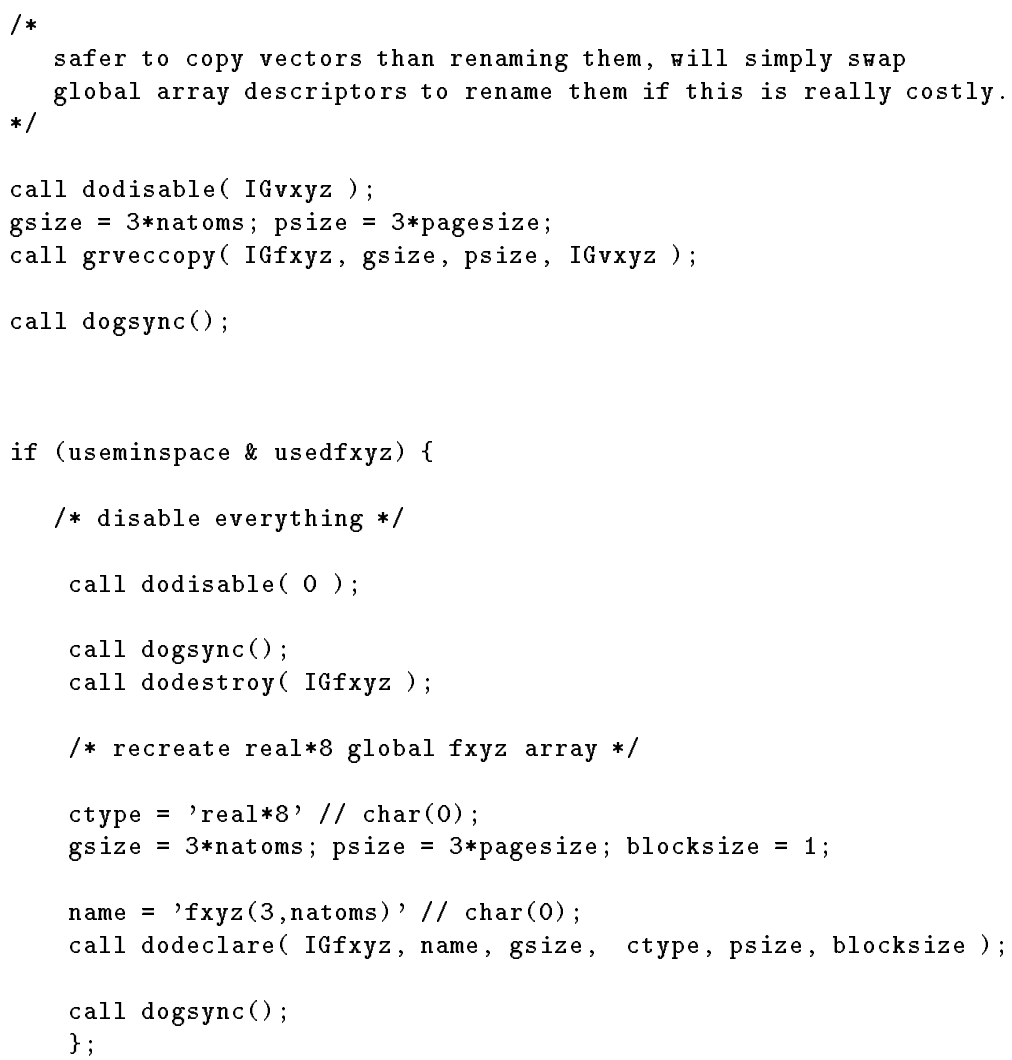

4.8. subroutine force

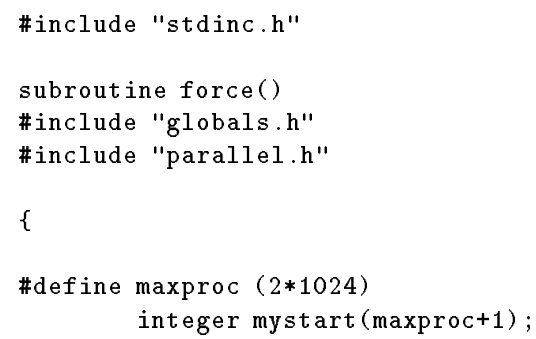


integer istrt,iend, ncols, binsize;

logical islast;

integer gsize,psize;

real one,zero;

parameter $($ one $=1.0$, zero $=0.0)$;

real8 oned,zerod;

parameter $($ oned $=1.0 \mathrm{~d} 0$, zerod $=0.0 \mathrm{~d} 0)$;

real rvalue;

real8 dvalue;

/* perform dynamic load balancing and all force computations */

$/ *========$ start execution $======* /$

\#ifdef USE_PROFILE

call profstart('force')

\#endif

ASSERT( ( $1<=$ nproc) \& (nproc <= maxproc),

, ** force: invalid nproc , nproc );

\#ifdef NO_BALAIICE

/* no need to call "caltork" */

call lbalance( mystart);

\#else

call profstart('force:calнork');

call calнork();

call lbalance( mystart);

call profend('force:calkork');

\#endif

istrt $=$ mystart $(m y i d+1) ;$

/* iend is the last column actually computed */

islast $=(($ myid +1$)==$ nproc $)$;

if (islast) \{

binsize $=$ nbiny $*$ nbinz:

iend = binsize;

\}

else \{

iend $=\operatorname{mystart}((\operatorname{myid}+1)+1)-1 ;$

\};

$n \operatorname{cols}=($ iend - istrt +1$)$

/* clear out array for accumulation */

gsize $=3 *$ natoms $;$ psize $=3 *$ pagesize $;$

\#ifdef USE_DFXYZ

dvalue $=0.0$

call gdvecfill( IGfxyz, dvalue, gsize, psize);

\#else

rvalue $=0.0 ;$

call grvecfill( IGfxyz, rvalue, gsize, psize);

\#endif 


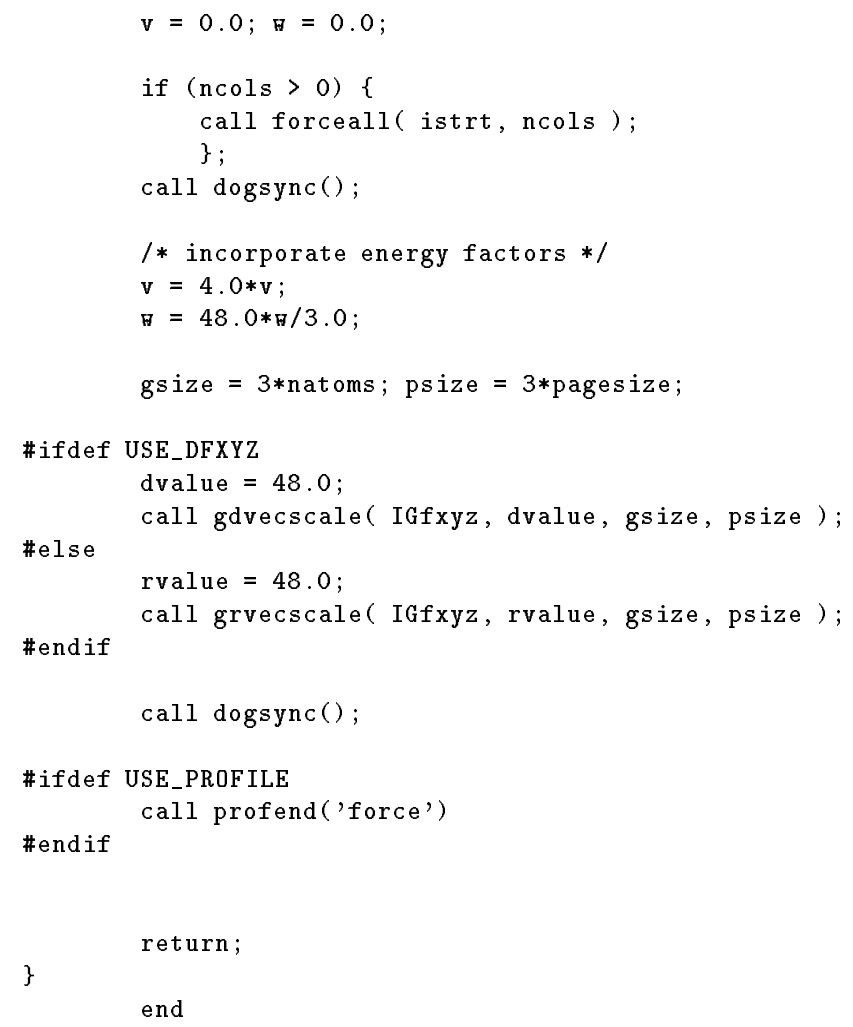

\section{9. subroutine forceall}

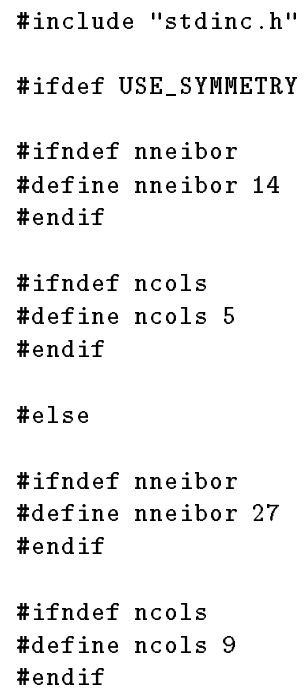




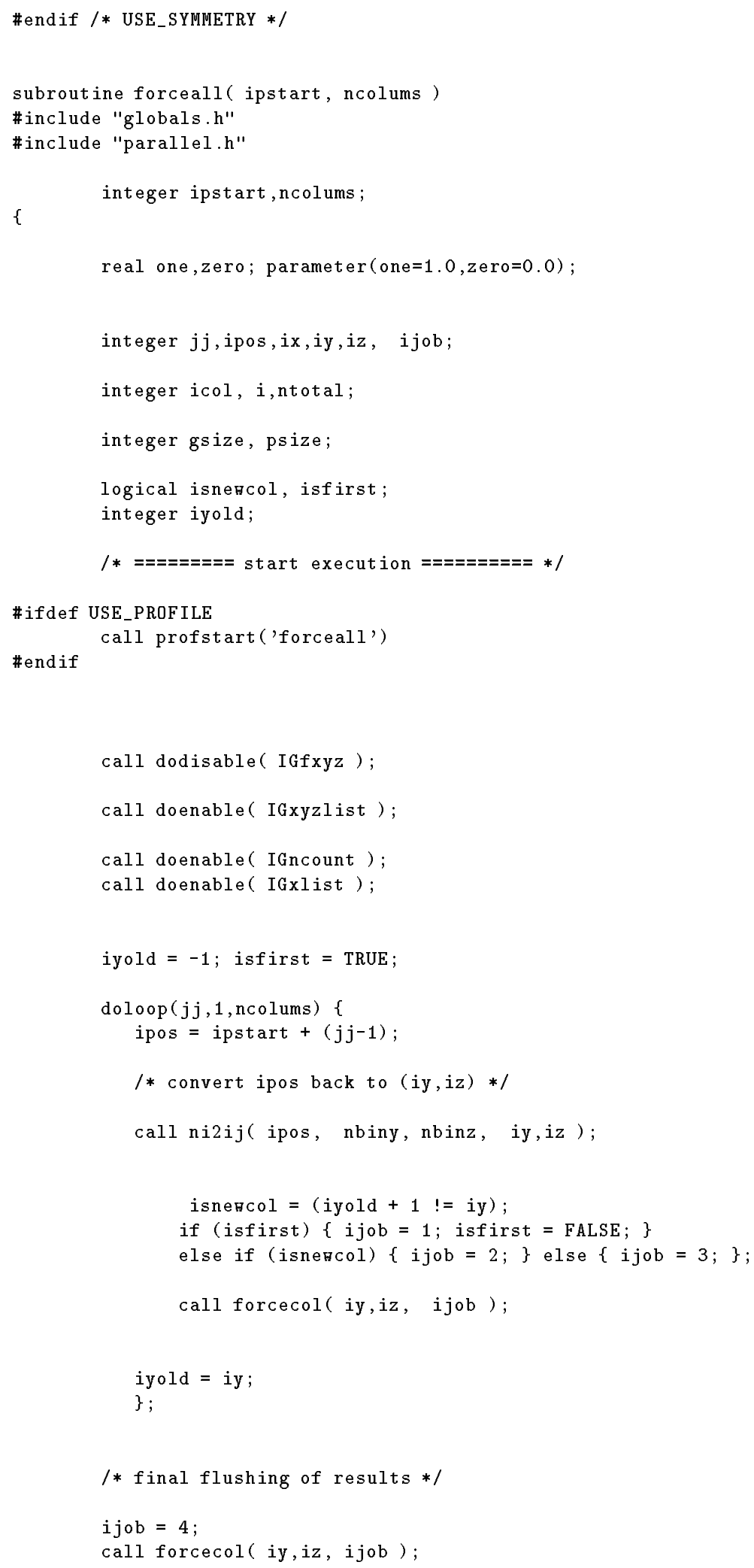




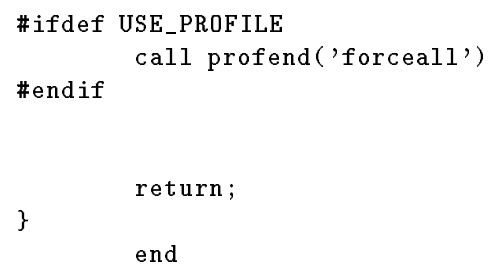

4.10. subroutine forcecol

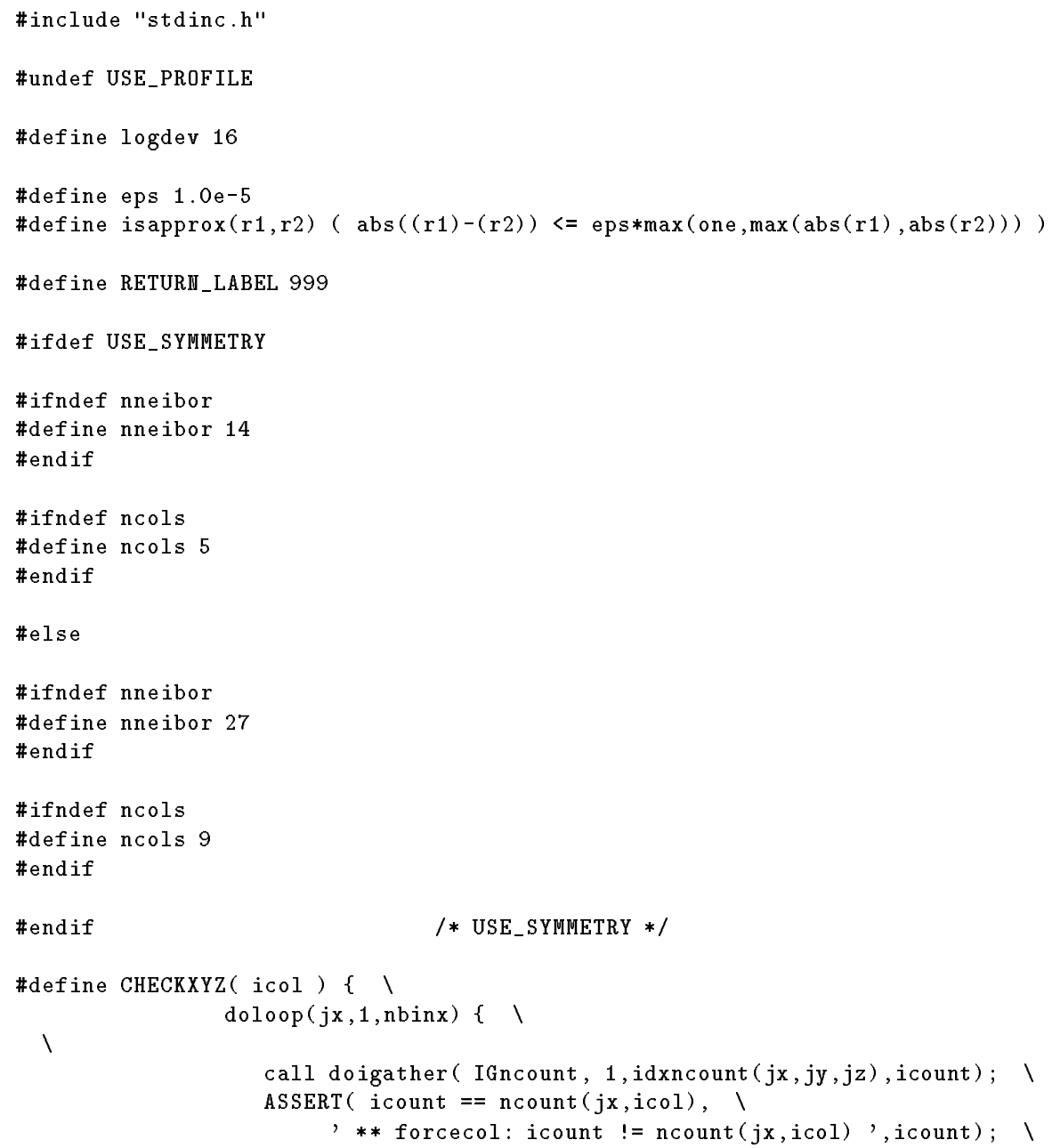




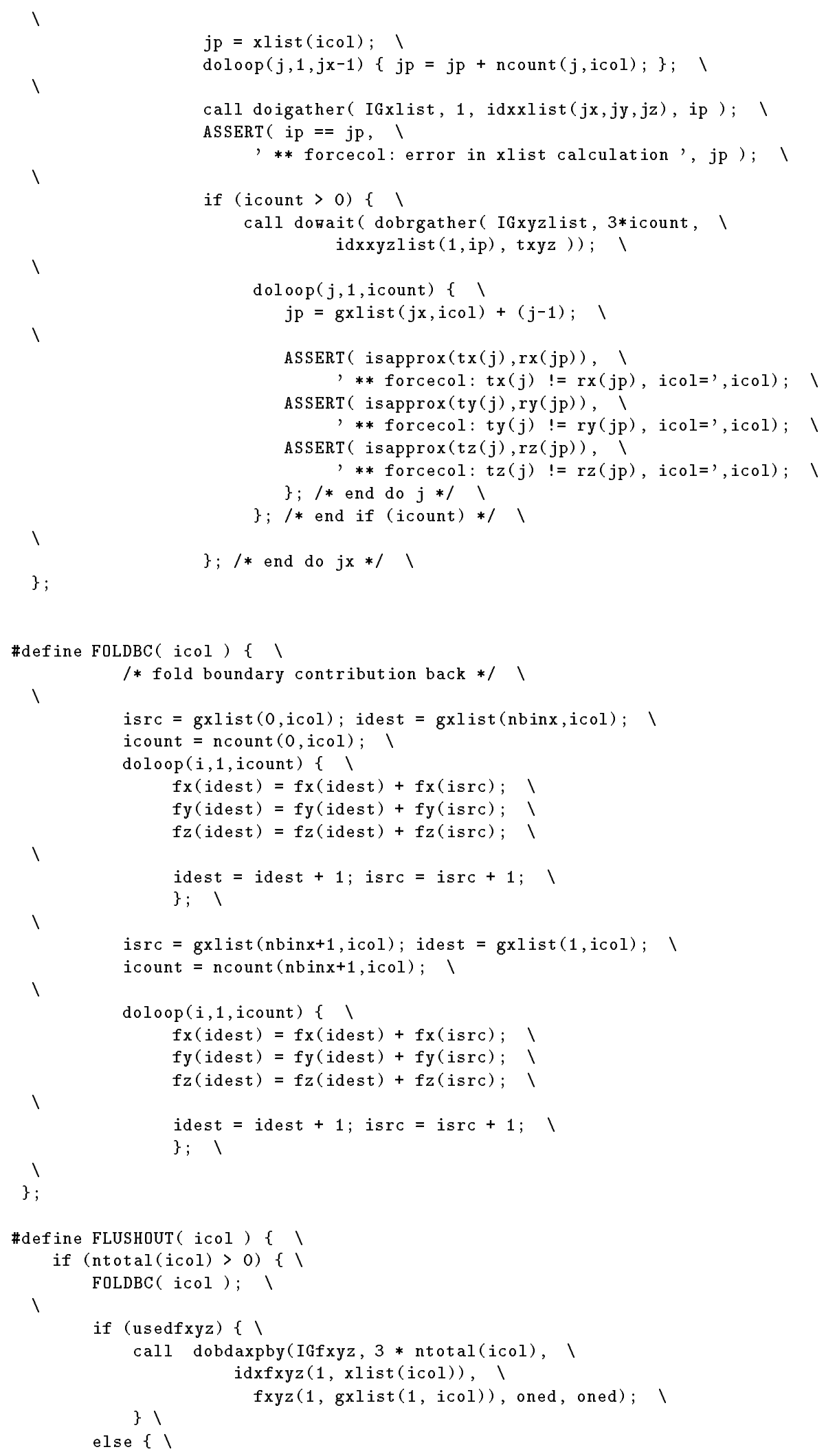




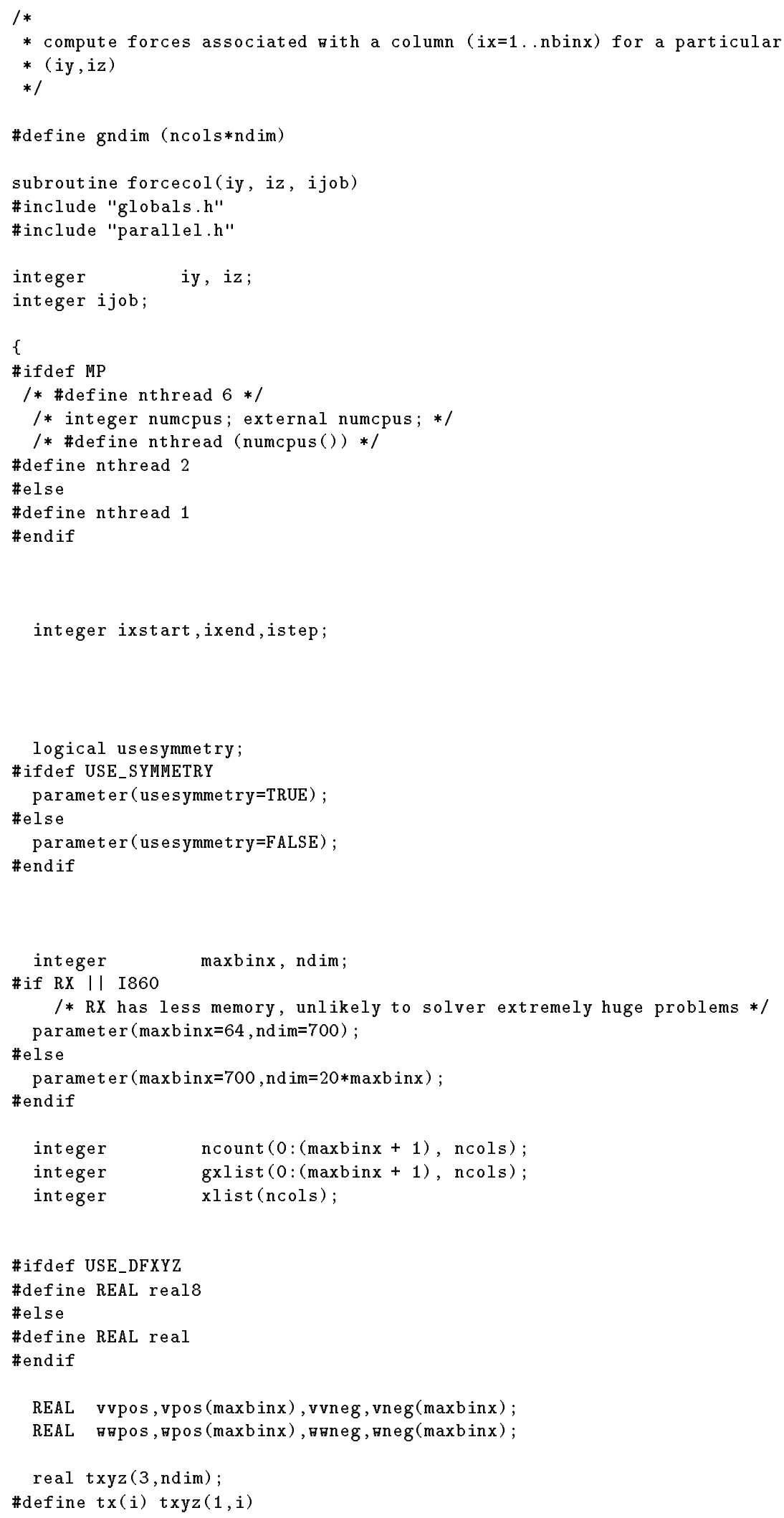




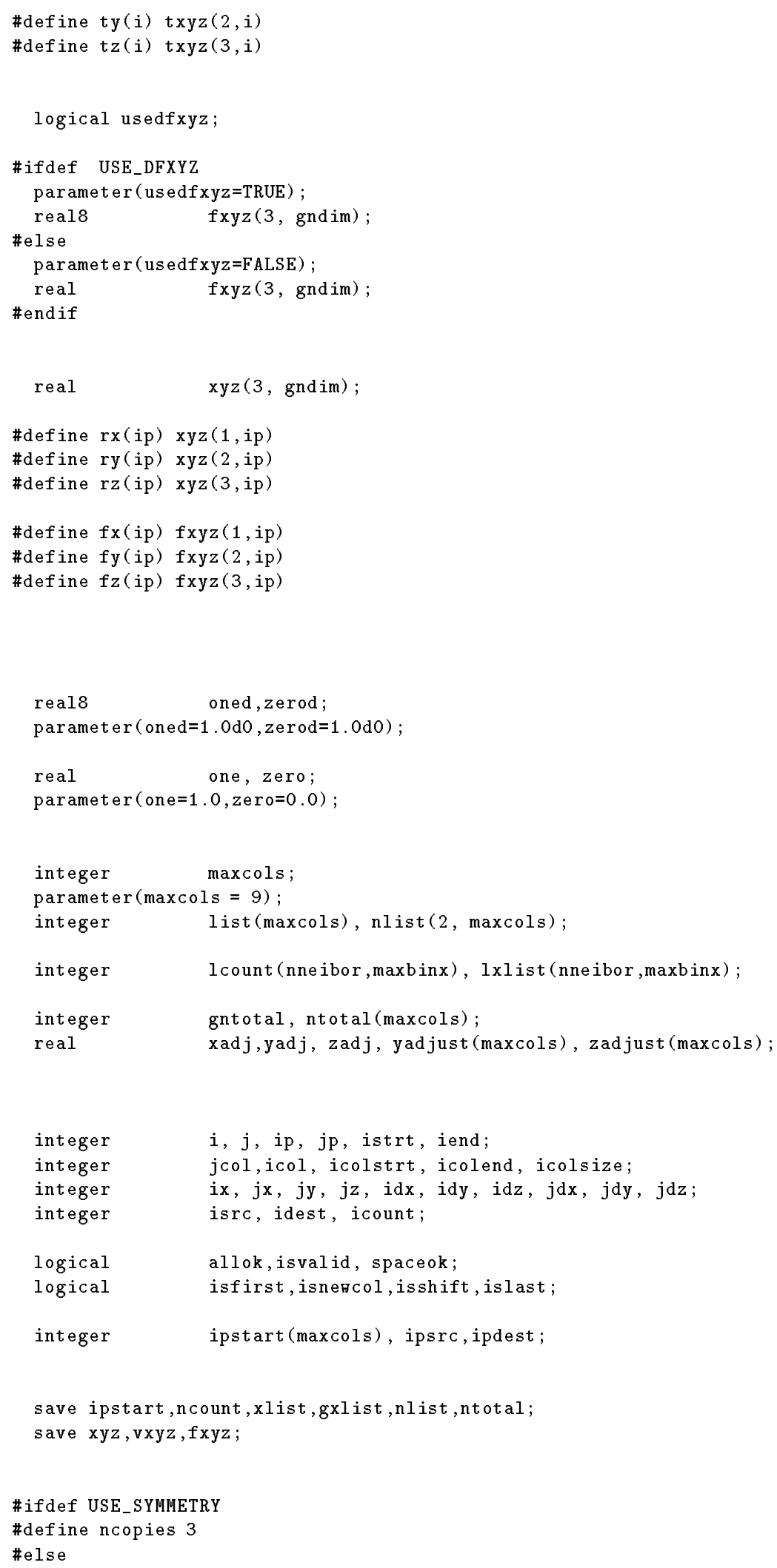


\#define ncopies 6

\#endif

integer nclist( 2 ,ncopies); save nclist;

\#include "mapping.h"

data isfirst / TRUE /;

\#ifdef USE_SYMMETRY

$/ *$ copy sequence $4->1,3->2$, then $5->3 * /$

/* based on the ordering:

$[*, *, 2]$

$[*, 1,3]$

$[*, 4,5]$

*/

data nclist / 4,1, 3,2, 5,3/;

\#else

$/ *$ copy sequence $4->1,5->2,6->3$, then

$7->4,8->5,9->6 * /$

/* based on the ordering:

$[1,2,3]$

$[4,5,6]$

*/

$[7,8,9]$

data nclist / 4,1, 5,2, 6,3 ,

$7,4,8,5,9,6 /$;

\#endif /*USE_SYMMETRY */

/* ==========" start execution =========== */

\#dir\$r noconcur

\#ifdef USE_PROFILE

call profstart ('forcecol');

\#endif

spaceok $=(1<=n$ binx $) \&(n b i n x<=\operatorname{maxbin} x)$;

ASSERT (spaceok,

, ** forcecol: invalid nbinx , nbinx);

isvalid $=(1<=$ ijob) \& (ijob $<=4)$;

ASSERT ( isvalid,

' ** forcecol: invalid ijob , ijob);

isfirst $=($ ijob $==1)$;

isneнcol $=(i j o b==2)$;

isshift $=(i j o b==3)$;

islast $=(i$ job $==4)$; 


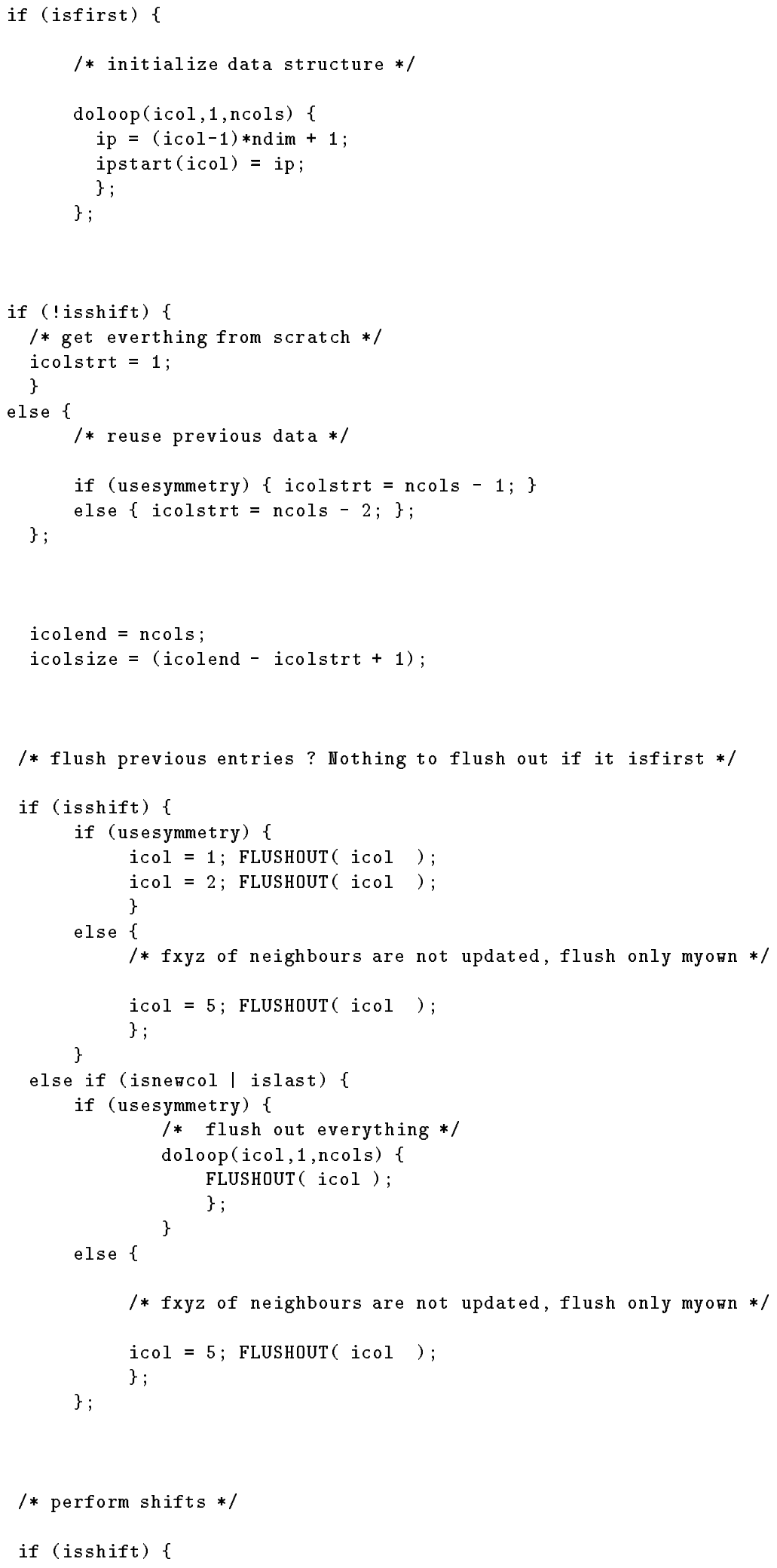




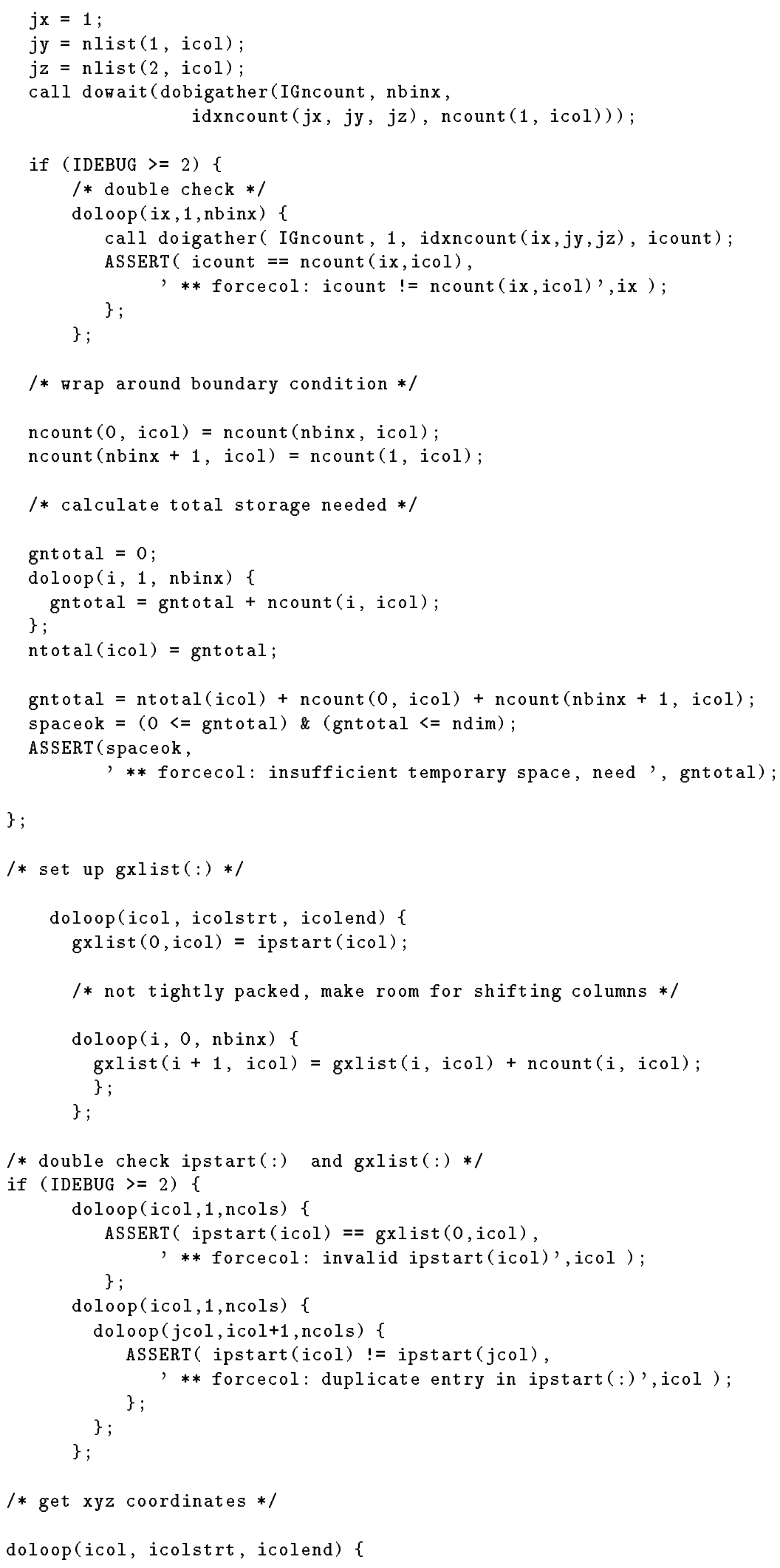




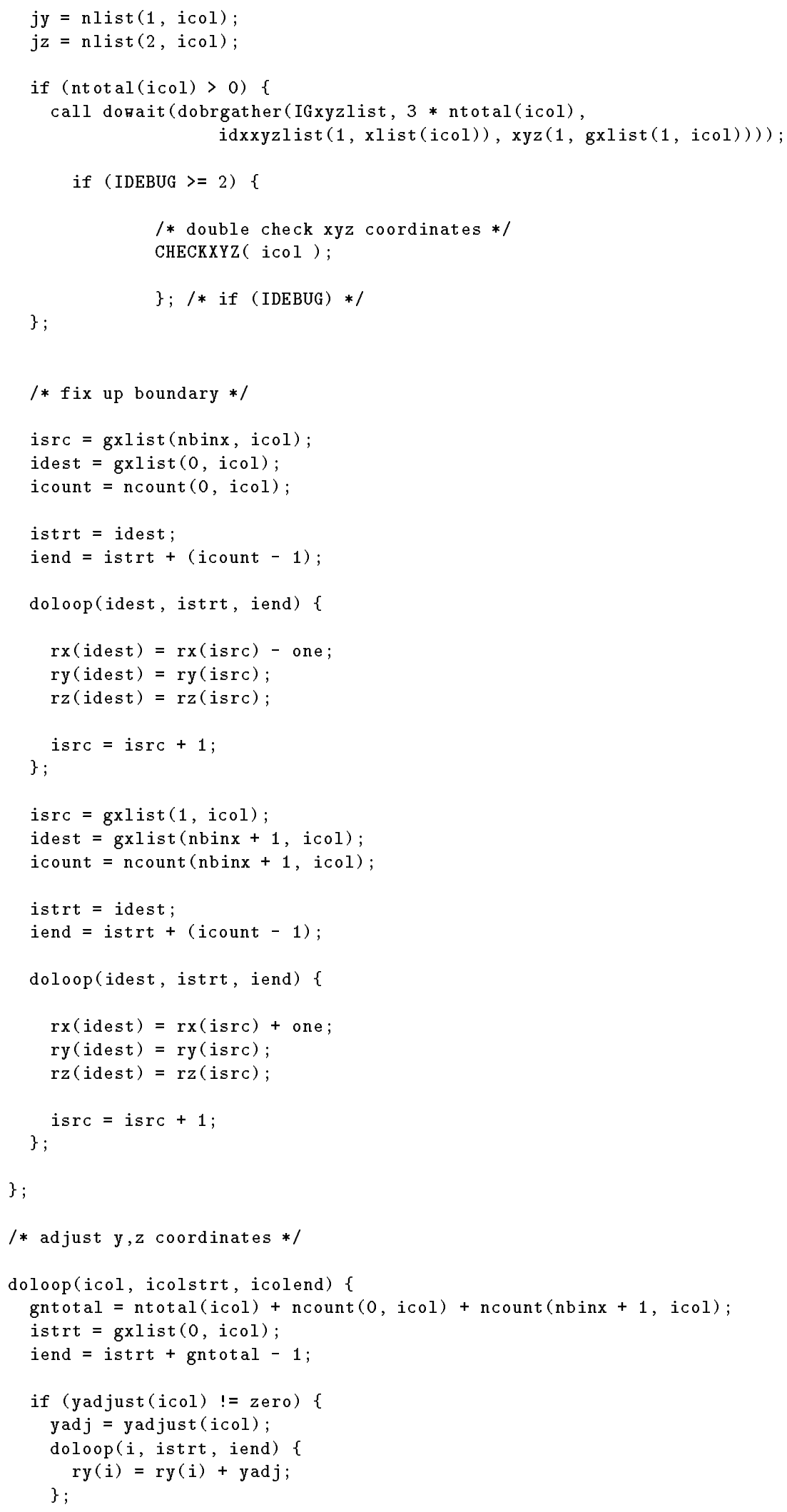




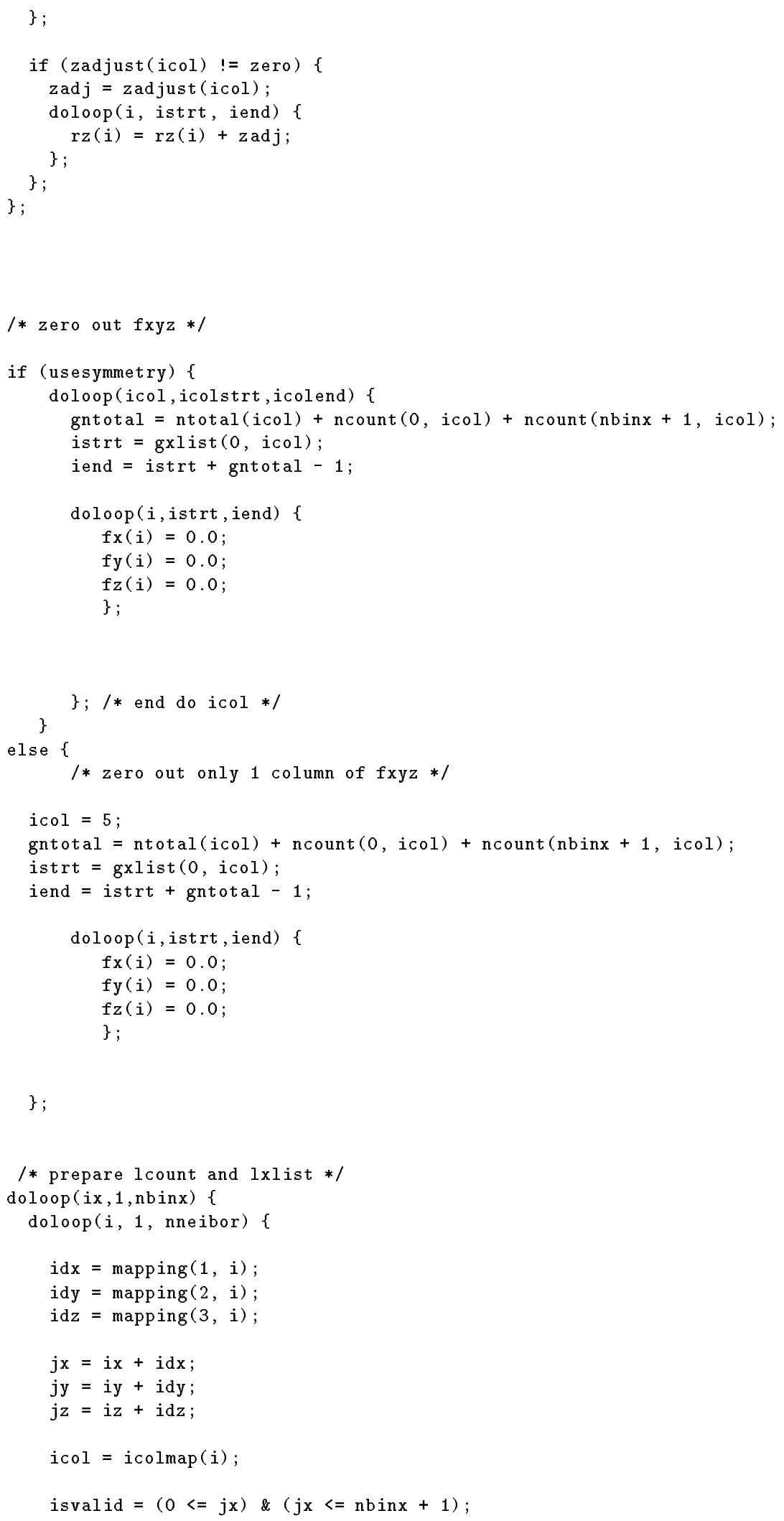




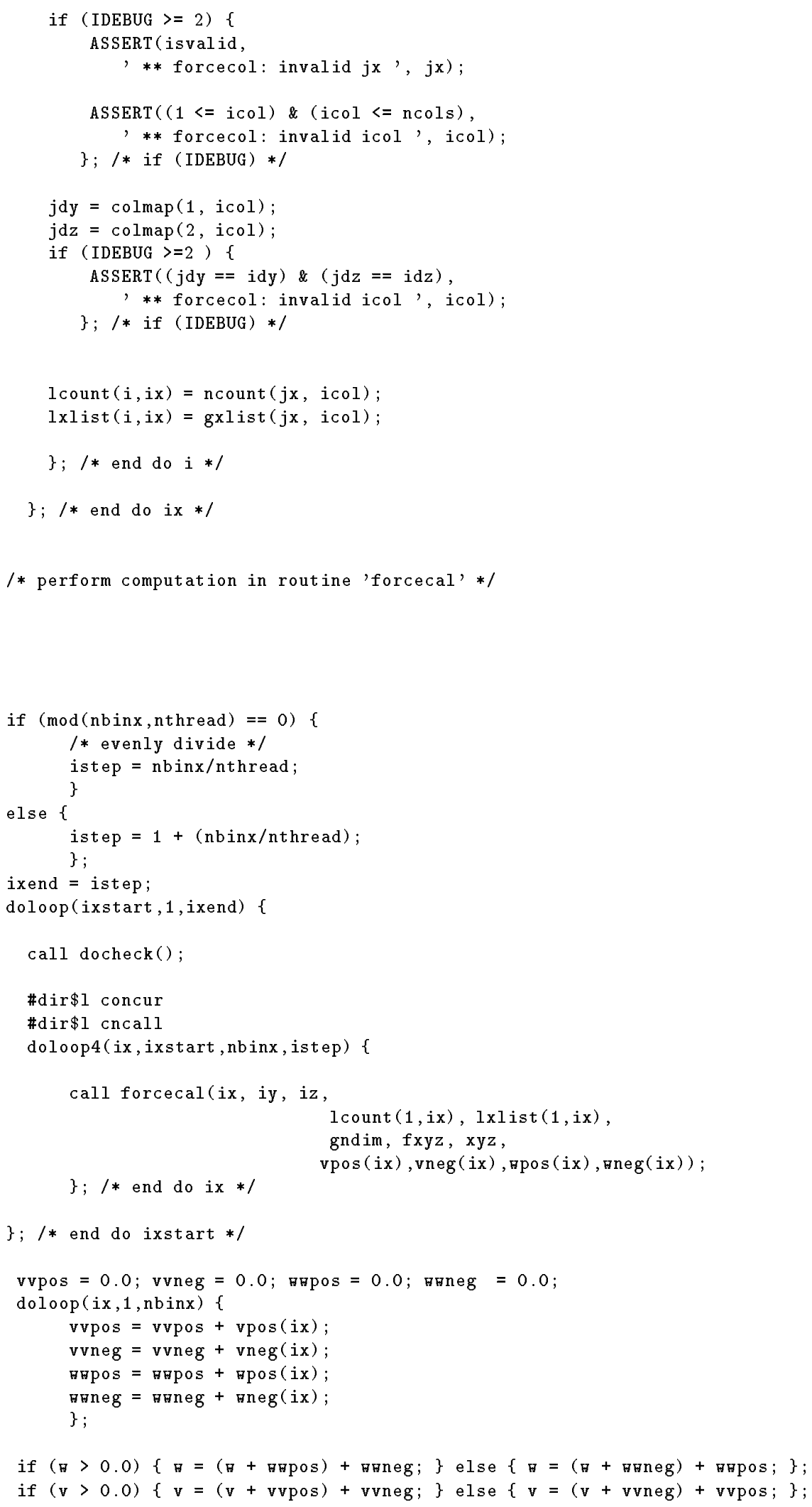




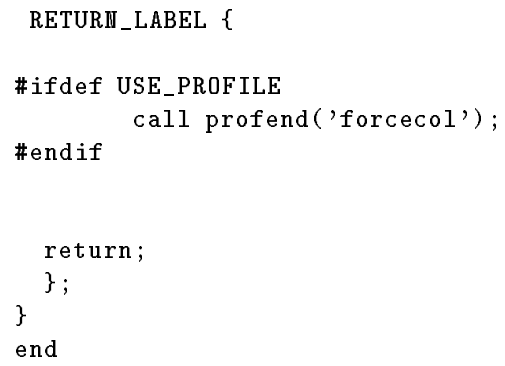

4.11. subroutine forcecal

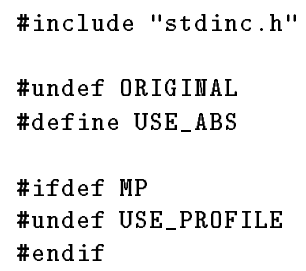




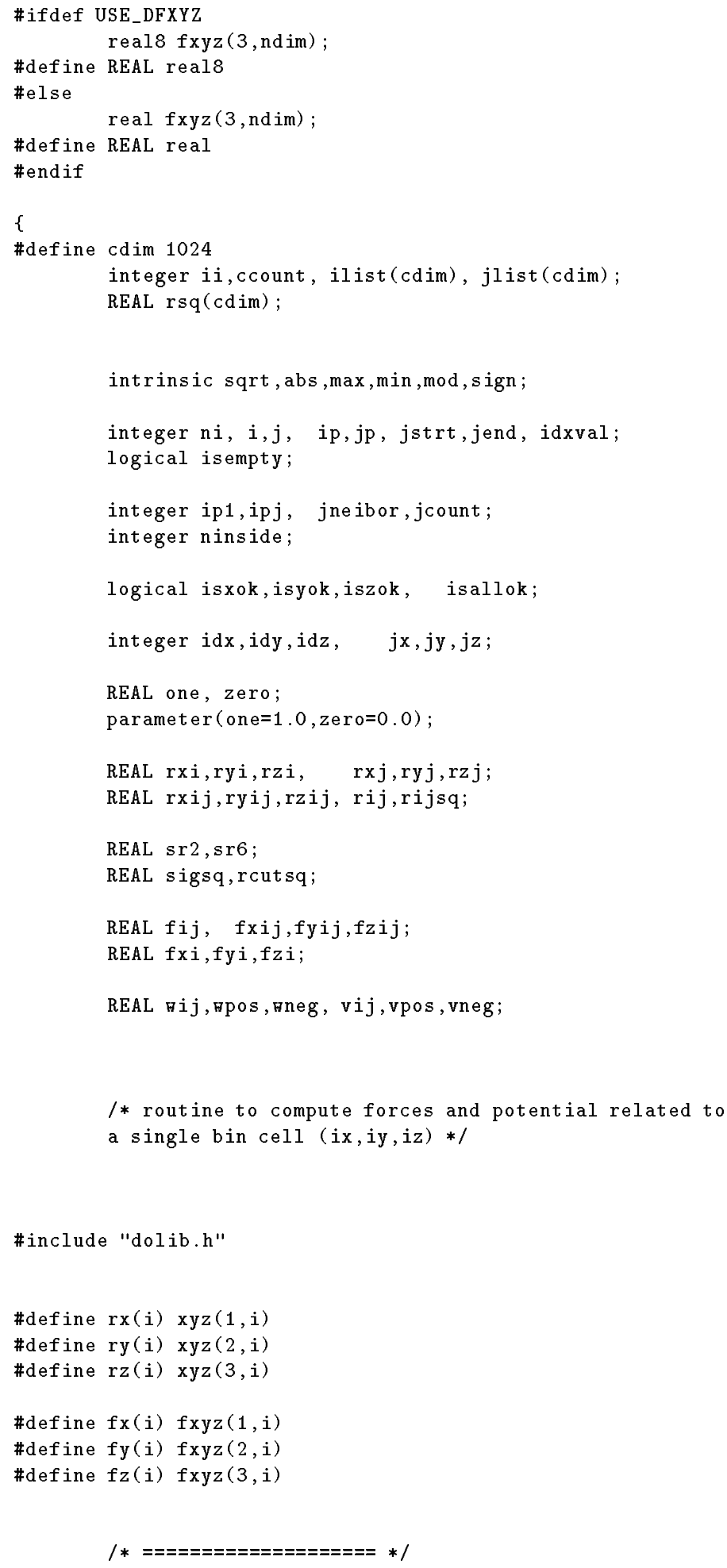




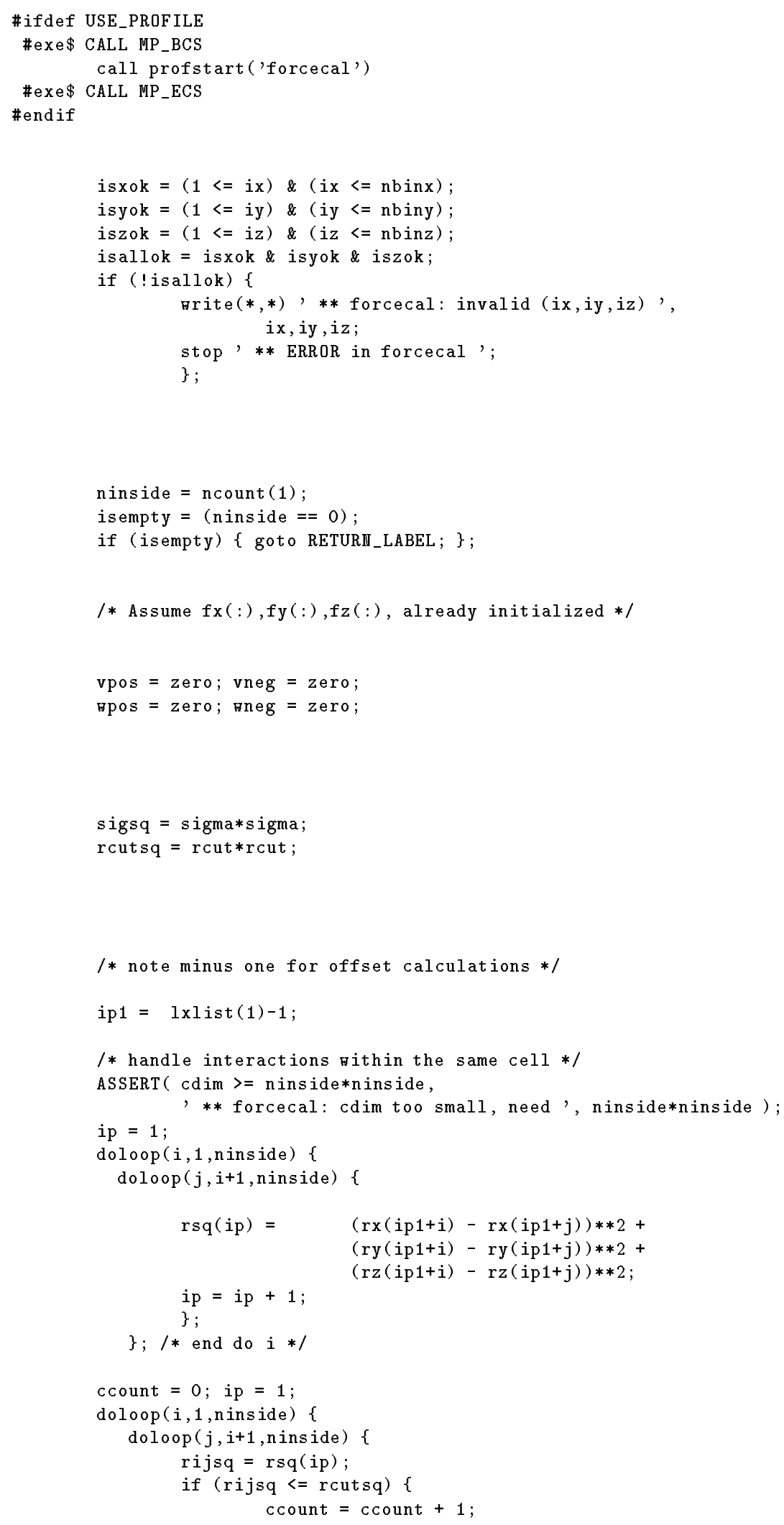




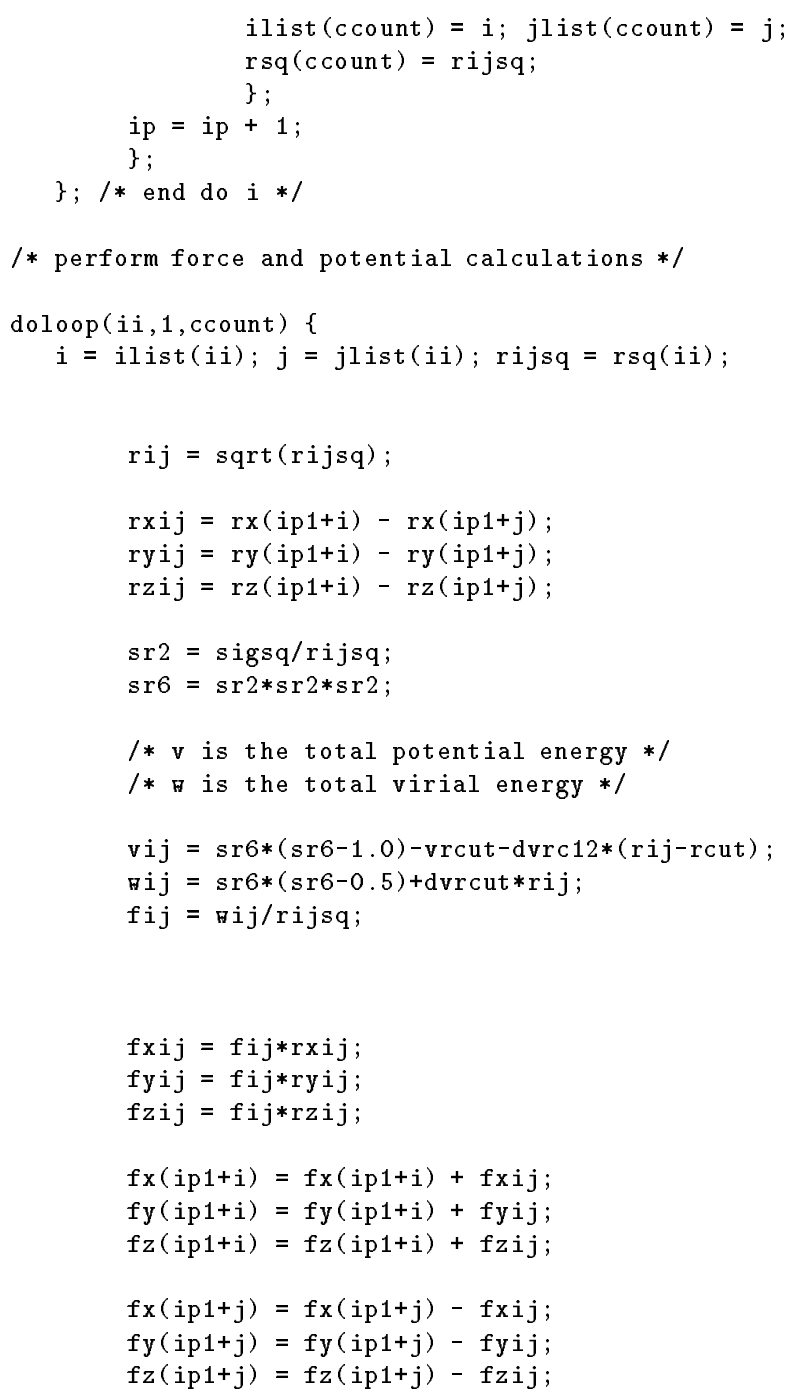




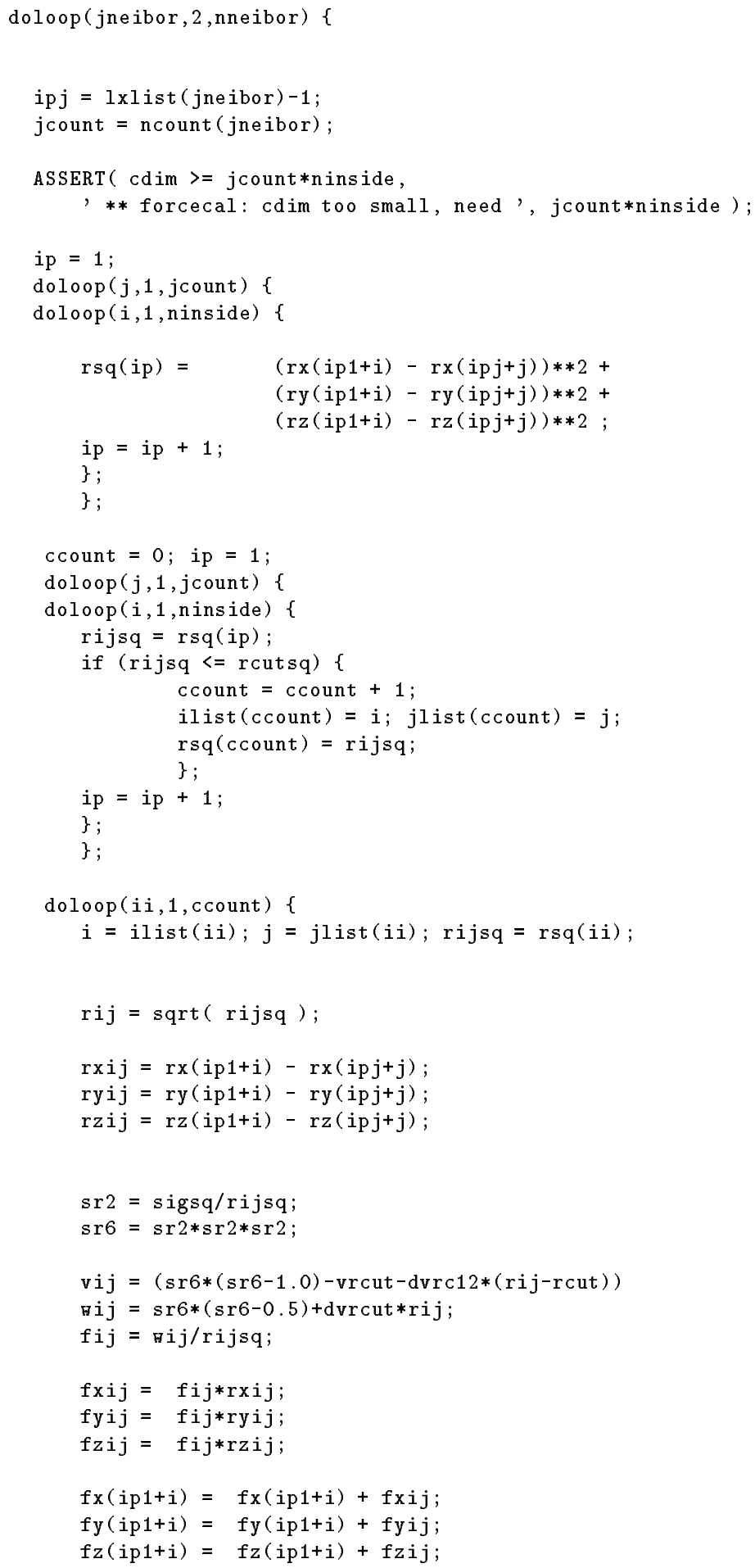


$f z(i p j+j)=f z(i p j+j)-f z i j ;$

\#else /*USE_SYMMETRY */

$$
\begin{aligned}
& v i j=0.5 * v i j \\
& \forall i j=0.5 * \text { *ij; }
\end{aligned}
$$

\#endif /*USE_SYMMETRY */

\#ifdef USE_ABS

$$
\begin{aligned}
& \text { vpos }=\operatorname{vpos}+(\operatorname{abs}(v i j)+v i j) ; \\
& \text { vneg }=\text { vneg }+(v i j-\operatorname{abs}(v i j)) ; \\
& \text { нров = нров }+(\operatorname{abs}(\text { нij) }+ \text { нij); } \\
& \text { нneg }=\text { нneg }+(\text { вij }-\operatorname{abs}(\text { нij)); }
\end{aligned}
$$

\#else$$
\text { if }(v i j>0.0)\{\text { vpos }=\text { vpos }+v i j ;\} \text { else }\{v n e g=v n e g+v i j ;\} \text {; }
$$$$
\text { if }(\text { нij > 0.0) \{нров }=\text { нров }+ \text { ніj;\} else }\{\text { нneg }=\text { нnеg }+ \text { ніj; }\} \text {; }
$$

\#endif /*USE_ABS */

\}; $/ *$ end do ii */

\}; /* end do jneigbor */

\#ifdef USE_ABS

vpos $=0.5 *$ vpos $;$ vneg $=0.5 *$ vneg;

нров $=0.5 *$ нров; нneg $=0.5 *$ нneg;

\#endif $/ *$ USE_ABS */

$/ *$ summation into global $\mathrm{v}$ and $*$ performed in forcecol */

RETURII_LABEL \{

\#ifdef USE_PROFILE

\#exe $\$$ CALL MP_BCS

call profend('forcecal')

\# exe\$ CALL MP_ECS

\#endif

return;

\};

\}

end 


\section{References}

[1] M. P. Allen And D. Tildesley, Computer Simulation of Liquids, Claredon Press, Oxford, 1987.

[2] D. M. BEasley and P. S. Lomdahl, Message passing multi-cell molecular dynamics on the Connection Machine 5, Parall. Comp., 20 (1994), pp. 173196.

[3] E. F. D'Azevedo And C. H. Romine, DoliB: Distributed Object Library, Tech. Report ORNL/TM-12744, Oak Ridge National Laboratory, 1994.

[4] R. W. Hockney, S. P. Goel, and J. W. Eastwood, Quiet highresolution computer models of a plasma, J. Comp. Phys, 14 (1974), pp. 148158.

[5] P. S. Lomdahl, P. Tamayo, N. Gronbech-Jensen, and D. M. BeaZLEY, 50 GFlops molecular dynamics on the Connection Machine 5, in Proceedings Supercomputing '93, November 15-19, Portland, Oregon, Assocation for Computing Machinery and The Institute of Electrical and Electronics Engineers, Inc., 1993, pp. 520-527.

[6] B. Marr, R. Peierls, and J. Pasciak, IPX - Preemptive remote procedure execution for concurrent applications, tech. report, Brookhaven National Laboratory, 1994.

[7] M. Pinches, D. Tildesley, And W. Smith, Large scale molecular dynamics on parallel computers using the link-cell algorithm., Molecular Simulation, 6 (1991), pp. 51-87. see also ftp://ftp.dl.ac.uk/ccp5/SOTON_PAR/README.

[8] S. Plimpton, Fast parallel algorithms for short-range molecular dynamics, Tech. Report SAND91-1144, UC-705, Sandia National Laboratories, Printed May 1993. 
[9] J. G. Powles, W. A. B. Evans, And N. Quirke, Non-destructive molecular dynamics simulation of the chemical potential of a fluid, Mol. Phys, 46 (1982), pp. 1347-1370.

[10] L. Verlet, Computer experiments on classical fluids I. Thermodynamical properties of Lennard-Jones molecules, Phys. Rev., 159 (1967), pp. 98-103. 